\title{
Über Mitwirkung der Plastosomen bei der Befruchtung des Eies von Filaria papillosa.
}

\author{
Von
}

Friedrich Meves in Kiel.

Hierzn Tafel I-IV.

\section{Einteilung.}

I. Einleitung . . . . . . . . . . . . . . . . . . . . 1?

II. Material and Methode . . . . . . . . . . . . . . . . . . 10

III. Die freien Spermien . . . . . . . . . . . . . . . . . . . . . Is

IV. Die unbefruchteten Eier and ihre Entwicklung. . . . . . . . . 21

V. Das eben eingedrungene Spermium . . . . . . . . . . . . . . 24

VI. Die Veranderungen am befmchteten Ei bis zur Bildung der beiden

Vorkerne . . . . . . . . . . . . . . . . . . . . . 27

VII. Der Beginn der Furchung . . . . . . . . . . . . . . . . . . :13

VIII. Schluss. . . . . . . . . . . . . . . . . . . . 38

\section{Einleitung.}

Wenn Pfeffer in der zweiten Auflage seiner I'flanzenphysiologie aus dem Jahre 1897 (S. 46) schreibt, dass für das Dogma, nach welchem der Kern der alleinige Träger der Erbmasse sei, ein zwingender Beweis überhaupt nicht erbracht worden ist. so hat dieser Satz noch heute gerade so gut wie damals Gültigkeit. "Es ist übrigens ganz unverkennbar", sagt Pfeffer an der zitierten Stelle weiter; "dass der Kern. welcher zuvor gar oft nebensächlich behandelt worden war, wesentlich durch die Beobachtung auffälliger formativer Vorgünge übermässig in den Vordergrund des Interesses und der Spekulation gerückt wurde. Die Degradation, welche der Kern nach der Entdeckung der Zentrosomen mehrfach erfuhr, indem er teilweise sogar nur zum dienenden Gliede herabgedrückt wurde, lehrt wiederum, in wie hohem Grade das Sichtbarwerden von Dingen die Deutung beeinflusst. Aller Wahrscheinlichkeit nach würde es a uch nicht an Theorien fehlen, welche dem Zytoplasma die Herrscherrolle zuweisen, wenn es fernerhingelingen sollte, in diesem aufallige Gestaltungenzu 
erspähen, die sich sicherlich im /yytoplasma abspielen, in welchem sich ebentalls die physiologischen Einheiten selbsttatig vermehren."

Solche "auffälligen Gestaltungen“ im Zytoplasma, welche meines Erachtens Vererbungsträger darstellen, haben wir inzwischen in den von mir sogenannten Plastosomen ") (früher Chondriosomen) genauer kennen gelernt; und ist es bereits in zwei Fällen, bei Ascaris (L. und R. $\mathrm{Zoja}^{3}$ ) Meves, Romeis, $\mathrm{Held}$ ) und Phallusia (Meves) gelungen, eine Beteiligung dieser Gebilde bei der Befruchtung nachzuweisen. Wir haben aber bisher niemals den Plastosomen die ganze Vererbung aufbürden wollen, sondern stets angenommen, dass Kern und Plastosomen. zusammen dabei wirksam sind.

Wenn zahlreiche Autoren an dem Vererbungsmonopol des Kerns festgebalten haben, solange eine Mitwirkung zytoplasmatischer Bestandteile des Spermiums bei der Befruchtung nicht direkt nachgewiesen war, so habe ich diesen Standpunkt völlig verstanden, wenn ich ihn auch schon vorher nicht geteilt habe. Dagegen habe ich die zahlreichen weiteren Hypothesen, mit welchen die O. Hertwig-Strasburgersche Lehre spater verquickt worden ist, meinerseits niemals als berechtigt anerkennen. könmen.

Zu diesen gehört zunächst die Van Beneden-RablBoverische Individualitätshypothese der Chromosomen, d. i. die Lehre, dass die Chromosomen selbständige Individuen sind, welche ibre Selbständigkeit auch im rubenden Kern

1) Von mir gesperrt.

2) $\mathrm{Zu}$ den Plastosomen gehören folgende Strukturelemente, von denen ich gezeigt habe, dass sie substantiell identisch sind: die Fila Flemmings von 1882, die Zytomikrosomen von v. Brunn und $v$. la ValetteSt. George, die Archoplasmakörner Boveris, die Bioblasten Altmanns, die Plastidulen der Gebrüder Zoja, die Mitochondrien Bendas etc. (vergl. Meves 1914,3 und 1915).

3) Die Gebrïder L. und R. Z oja haben schon 1891 beschrieben, dass bei der Befruchtung von Ascaris die "Plastidulen" des Spermiums sich mit denjenigen des Eies vermischen. Sie haben aber in theoretischer Hinsicht. ihrem Befund keinen Wert beigelegt. Der eine der beiden Brüder, $R . Z \circ j a$, hat 6 Jahre später (1897, S. 17) direkt ausgesprochen, dass das Protoplasma des Sperminms bei der Vererbung keine Rolle zu spielen und, $a \mathrm{uch}$ bei As caris, vom Eikörper resorbiert zu werden scheine. 
bewahren. ${ }^{1}$ ) Ich habe schon bei mehreren Gelegenheiten (1907; 1908,2 ; 1911,2) meinen Unglauben daran bekannt, welchen ich übrigens mit verschiedenen anderen Autoren, O. Hertwig (1890), Flemming $\left.{ }^{2}\right)$ (1894), Jost (1904), Fick (1905-1908), I ussbaum (1906), v. Tellyesniczky (1907), Della Valle (1909) u. a. teile.

Nach Boveri wird man $z u$ dieser Hypothese in exster Linie durch die Tatsache genötigt, dass bei der Zellteilung Chromosomen von konstanter Zahl und Grösse aus dem ruhenden Kern hervorgehen. Demgegenüber ist folgendes $\mathrm{zu}$ bemerken (vergl. Meves 1911,2 S. 296): In den Spermatozyten zahlreicher wirbelloser Tiere, z. B. denjenigen der eupyrenen Generation von Paludina, bilden sich im Beginn der ersten Reifungsteilung aus der Masse der Plastochondrien Stäbe, Plastokonten, von bestimmter Länge und sehr wahrscheinlich auch Zabl. In den Spermatiden derselben Zellgeneration von Paludina entsteben aus diesen Plastokonten vier gleichgrosse Fïgelchen oder Blaschen, welche sich um die Ansatzstelle des Schwanzes herum dem Kiern anlagern. In den Spermatozyten von Paludina, welche den oligopyrenen Spermien Entstehung geben, zerfallen die beiden Zentriolen im Beginn der ersten Reifungsteilung jedes in $z w o ̈ l f$ gleich grosse Körner (Meves 1903). Bei den angeführten Beispielen, denen sich leicht noch andere ahnliche anreihen liessen, handelt es sich um Bildungen, welche ebenso wie die Chromosomen völlig selbständig sind und wie diese in konstanter Grösse und Zahl auftreten, von denen es aber ausgeschlossen ist, dass sie im individualisierten Zustand vorler existiert haben. Sie ent-

1) Nach Boveri ist das Chromosom im Ruhekern nacl Art eines Rhizopoden in ein Gerüstwerlk übergegangen; im Beginn der Mitose zieht es sich wieder zusammen.

2) Flemming hat sich, so viel ich weiss, öffentlich nur ein einziges Mal, an einer Stelle, die ich erst külzlich autgefunden habe, gegen die Individualitätshypothese ausgesprochen. In einem 1894 erschienenen Bericht über Morphologie der Zelle in Bd. 3 der Merkel-Bonnetschen Ergebnisse der Anatomie und Entwicklungsgeschichte sagt er S. 111, Anm.1, dass wir durch die Arbeiten Rü ckerts, Borns u. a. wüssten, dass bei manchen Ovarialeiern die Chromosomen schon während der Eireifung individualisiert angelegt werden und in diesem Zustand sehr lange bestehen können. „Allgemeine Geltung für die Eizelle", fährt er fort, scheint dies nicht $z \mathfrak{u}$ haben, geschweige denn für andere Zellarten." 
stehen vielmelnr neu aus einem bestimmten, in der Zelle vorhandenen Material. Das Bedingtsein ihres Entstehens aber ist uns absolut dunkel. Das gleiche gilt meines Erachtens für die Chromosomen.

Auch von den weiteren Argumenten, welche Boveri zugunsten der Individualitätshypothese anfülurt, kann ich keinen als stichhaltig anerkennen (vergl. Meves, 1911.2).

Die Individualitütshypothese macht für die Reifungsteilungen die weitere Annahme nötig. dass im Beginn derselben je zwei Chromosomen zusammentreten, erweist sich aber auch dadurch als unrichtig, dass die zahlreichen, im Glauben an die Individualitätshypothese unternommenen Versuche, eine solche "Konjugation" individualisierter Chromosomen zu erweisen, sämtlich gescheitert sind. Fon allen Beobachtungen, welche als Beweis für eine Konjugation angeführt werden, lässt sich meines Erachtens unschwer zeigen, dass sie irrtümlich gedeutet sind (vergl. Weves 1907, 1908,2). Dass diesen Beobachtungen keine Beweiskraft innewohnt, geht auch für den Uneingeweihten daraus hervor, dass die Anhänger der Konjugation unter sich nicht einmal über die Art derselben, ob sie eine „endweise" oder „parallele" sei, einig sind.

Boveri selbst hat übrigens neuerdings die Bildung der Chromosomen mit einer Vorstellung vereinbar gefunden, welche einem Verzicht auf die Individualitatshypothese gleichkommt (vergl. Meves 1911,2, S. 295).

An die Individualitäts- und Konjugationshypothese schliessen sich noch verschiedene andere Chromosomenhypothesen an, welche sogar in noch höherem Maße anfechtbar sind, welche aber nichtsdestoweniger zusammen mit den erstgenannten in zahlreichen Abhandlungen und sogar in Lehrbüchern als bewiesen angenommen werden und vielfach als Basis fül weitgehende Spekulationen dienen müssen.

In der vorliegenden Arbeit habe ich ein weiteres Beispiel für eine Beteiligung der plastosomatischen Substanz des Spermiums bei der Befruchtung mitzuteilen.

\section{Material und Methode.}

Als ich im August des Jahres 1913 die Gastfreundschaft der schwedischen Zoologischen Station Kristineberg genoss, traf ich eines Tages im Aquarium Herrn Prof. L. Jägerskiöld 
damit beschäftigt, eine grössere Anzahl Filarien (Filaria spirocauda Leidy), welche er soeben im rechten Herzen eines von ihm erbeuteten Seehundes (Phoca vitulina L.) aufgefunden hatte, zu konservieren. An einem mir freundlichst überlassenen Exemplar. welches sich als Weibchen erwies, untersuchte ich den Inlalt der Uterusschläuche in frischem Zustand und gewann dabei auf Grund der Kleinheit, Dünnschaligkeit und Durchsichtigkeit der Eier den Eindruck, dass sie ein für zellulare Studien sehr geeignetes Objekt bilden müssten. Dadurch wurde der Wunsch in mir rege, den Befruchtungsvorgang bei Filaria mit Hilfe der Plastosomenmethoden zu untersuchen. Herr Prof. Jägerskiöld hielt einen Versuch zur Beschaffung voll weiterem Material nicht für aussichtslos und erbot sich liebenswürdigerweise, mir dabei behülflich zu sein. Jedoch kam die von uns geplante Seehundsjagd wegen schlechten Wetters, welches bis zu meiner Abreise von Kristineberg andauerte, nicht zur Ausführung.

Mein Interesse für die Befruchtung des Filariaeies war auch nach meiner Rückkehr nach Kiel im September 1913 lebendig geblieben. Ich nahm daher die Schneidersche „Monographie der Nematoden" zur Hand, um mich über das Vorkommen von Filarien $\mathrm{zu}$ orientieren, und fand darin eine in der Bauchhöhle des Pferdes lebende Filarie, Filaria papillosa R., bei welcher das Weibchen bis zu $110 \mathrm{~mm}$ lang wird, als „leicht zugängliches Objekt" bezeichnet. Daraufhin setzte ich mich mit dem hiesigen Schlachthof in Verbindung, und gelang es mir, in der Zeit von Anfang Oktober 1913 bis Ende Juli 1914, also innerhalb von 10 Monaten, ${ }^{1}$ ) $30-35$ Exemplare von Filaria papillosa (in $7-8$ Lieferungen) zu bekommen.

Die Auffindung der Würmer auf dem Schlachthof gestaltete sich folgendermaßen: Bei Ausführung der Schlachtung wird das getötete Pferd an den Hinterbeinen aufgehängt und hochgewunden und dann die Bauchhöhle durch einen Schnitt in der Mittellinie der Bauchwand eröffinet; darauf werden die Eingeweide ausgeräumt, wobei sie nach der hier in Kiel (und wohl auch anderswo) geübten Methode auf einer Karre aufgefangen werden. Vorhandene Filarien findet man zwischen den Eingeweiden oder am Boden der Karre umherkriechen.

2) Innerhalb dieser Zeit wurden in Kiel ca. 750 Pferde geschlachtet. 
Die aufgefundenen Würmer wurden in ein Thermophor gebracht, welches vorher (zur Erzeugung einer höberen Temperatur im Innern) mit warmem Wasser ausgespült und zur Hälfte mit warmem Pferdemist gefüllt wurde, und dann ohne irgendwelchen Verzug in das Anatomische Institut transportiert; sie kamen stets lebend (nach Versicherung des Schlachters in allen Fällen ungefähr eine Stunde nach dem Tode des Wirts) in meine Hände.

Sümtliche mir überbrachten Würmer waren Weibchen; in einem Fall, in dem nur zwei Würmer gefunden waren, erwiesen sich diese später als unbefruchtet.

Die weiblichen Geschlechtsorgane von Filaria papillosa sind zweiteilig; die beiden Äste des Uterus gehen nach sehr langem Verlauf an ihrem hinteren Ende mit einem Absatz in die kurzen feinen Eileiter über, welche sich ihrerseits in die bis etwa $4 \mathrm{~cm}$ langen ()varien fortsetzen. Filaria papillosa gehört $z u$ den viviparen Nematoden. Der grösste Teil der Uterusschläuche ist mit gefurchten Eiern und jungen Würmein gefüllt. Für das Studium der Befruchtung und ersten Furchung kommen nur die hintersten, $3-4 \mathrm{~cm}$ langen Stücke der Uterusschläuche in Betracht. Um diese zu gewinnen, verfahre ich folgendermaßen. Ich bringe den Wurm in eine mit Wachs ausgegossene Schale, deren Boden mit warmer physiologischer Kochsalzlösung bedeckt ist, stecke Kopfund Schwanzende fest, spalte, vom Kopfende anfangend, den Hautmuskelschlauch in ganzer Länge, ${ }^{1}$ ) schlage die Spaltränder nach aussen um und fixiere sie mit Nadeln. Jann entwirre ich die Uterusschläuche, durchtrenne sie ca. $5-6 \mathrm{~cm}$ vor dem Übergang in die Eileiter und bringe die hinteren Abschnitte mit den Eileitern und Ovarien in die Fixierungsflüssigkeit hinein.

Für die Fixierung habe ich zuerst Altm a n nsches Gemisch gebraucht, habe aber gefunden, dass es bei diesem Objekt eine nicht unerhebliche Schrumpfung hervorruft. Gute Resultate erzielte ich dagegen mit der Flem mingschen Chromosmiumessigsäure, welche ich in der von mir 1908,1 für Plastosomenstudien empfohlenen Zusammensetzung anwandte; das auf diese Weise fixierte Material habe ich hinterher vielfach noch nach Benda nachbehandelt, indem ich es zunächst auf 24 Stunden in ein Gemisch von Holzessig und 1 proz. Chromsäure und dann auf

1) Hierbei bediene ich mich eines $G \mathrm{r}$ a ef eschen gebogenen Zystotoms, wie es in der Augenheilkunde zur Spaltung der Linsenkapsel gebraucht wird. 
weitere 24 Stunden in eine 2 proz. Lösung von Kalium bichromicum hineinbrachte. Von weiteren Fixierungsmitteln, welche zum Studium der Plastosomen gebraucht werden, habe ich noch die von $\mathrm{L}$ evi (1913) empfohlene Modifikation des Gemisches von Maximow geprüft, habe aber gefunden. dass sie bei den Filariaeiern nicht so gutes wie die modifizierte Flem ming sche Flüssigkeit leistet. Schliesslich habe ich noch einen allerdings nur kleinen Teil meines Materials (die hinteren Enden der Geschlechtsröhren von je zwei Würmeln) mit Sublimat-Alkohol-Eisessig ${ }^{1}$ ) nach v. Le n bo ss ék und mit Pikrinsäure-Formol-Eisessig ${ }^{2}$ ) nach bouin tixiert.

Nachdem die Geschlechtsröhren die erforderliche Weiterbehandlung durchgemacht hatten, wurden sie, vor der l̈bertragung in Xylol, in ca. $1 \mathrm{~cm}$ lange Stücke zerteilt; diese wurden zu mehreren in Paraffin eingebettet und der Lange nach in $4 \mu$ oder 5 "l dicke Schnitte zerlegt.

Zur Fürbung des mit dem modifizierten Flemming schen Gemisch fixierten Vaterials habe ich in erster Linie die Eisenhämatoxylinmethode, meistens mit Torbehandlung nach Rubaschkin, angewandt. Auch mit der Bendaschen Eisenalizarin-Kristallviolettfärbung habe ich eine Reihe von Versuchen angestellt; jedoch ist mir eine Darstellung der kleinen Eiplastochondrien auf diese Weise nicht gelungen. Ausserdem habe ich noch eine Anzahl Fürbungen (s. u.) probiert, um die schwer nachweisbare Kernsubstanz des eingedrungenen Spermiums zu verfolgen; dies glüclite mir an dem mit Sublimat-Alkohol-Eisessig belıandelten Waterial mit Hülfe der Giemsa schen Azur-Eosinfärbung, welche ich nach der Vorschrift anwandte, die Giemsa selbst (Deutsche medizinische Wochenschrift, Jahrg. 36, 1910) für die Schnittfürbung gegeben hat.

III. Die freien Spermien. (Fig. 1-10 und 39-48.)

Die freien Spermien habe ich an Schnitten durch das oberste Uterusende studiert. welches in den meisten Fallen auf eine kleinere oder grössere Strecke (häufig auf nicht weniger als $6-8 \mathrm{~mm}$ ) damit erfüllt ist.

1) Konzentrierte wässerige Sublimatlösung $75 \mathrm{ccm}$, Alkohol abs. $25 \mathrm{ccm}$. Eisessig $5 \mathrm{ccm}$.

2) Gesättigte wässerige Pikrinsäurelösung 7ó ccm, Formol $25 \mathrm{ccm}$. Eisessig $\tilde{a} \mathrm{ccm}$. 
Die Spermien sind rundliche oder länglich rundliche Zellen, welche an Insektenspermatiden erinnern, die auf einem frühen Stadium der Entwicklung (unmittelbar nach Ablauf der zweiten Reifungsteilung) stehen geblieben sind. Bei Fixierung mit Flem ming schem Gemisch und Fürbung mit Safranin (Fig. 1-5) zeigen sie einen Zytoplasmaleib, welcher ausser der Kernsubstanz einen grossen, plastosomatischen "Nebenkern" einschliesst, wie wir ihn zuerst bei Insektenspermatiden kennen gelernt liaben (vergl. Wheves, 1900). Die Kernsubstanz liegt am vorderen Pol der Samenzelle in einer zytoplasmatischen Kappe, welche den gleichfalls vorn gelegenen Nebenkern bedeckt. Diese Kappe scheint resistenter zu sein als das Zytoplasma des Schwanzteils; denn wilhrend letzteres an dem mit Fle mm ing schem Gemisch fixierten Material nicht selten zertlossen erscheint, ist die Kopfliappe stets woln erhalten. Die Kermsubstanz liegt nicht immer genau am vorderen Pol des Spermiums vor dem Nebenkern, sondern vielfach an der Seite desselben, so dass sie bei stark gefürbtem Nebenkern von diesem völlig verdeckt wird. Sie bildet keinen einheitlichen Körper, sondern wird durch eine Gruppe kleiner Kü̈gelchen repräsentiert, welche mitunter mehr oder weniger stark miteinander verbacken erscheinen, in den meisten Fallen aber völlig voneinander isoliert sind; man kann sie dann zählen und feststellen, dass bald fünf (Fig. 1, 4, 5), bald sechs (Fig. 2, 3) solcher Kügelchen vorhanden sind.

Der gleiche interessante Befund in bezug auf die. Kernsubstanz ist von Mulsow $(1911,1912)$ bei den Spermien eines verwandten Nematoden, Ancyracanthus cystidicola, welcher in der Schwimmblase der Forelle lebt, erboben worden. Mu lsow kommt durch Untersuchung der Spermatogenese zu dem Resultat; dass die Kügelchen als die Chromosomen der zweiten Reifungsteilung aufzufassen sind, welche getrennt nebeneinander liegen bleiben. Fon den sechs Chromosomen, welche bei der Hälfte der Spermien vorhanden sind, ist eines als Geschlechts- oder Heterochromosom aufzufassen. In den Prophasen der ersten Reifungsteilung zühlt man sechs Chromosomen, von denen sich eines durch geringere Grösse auszeichnet. Dieses, das Heterochromosom, gelangt später ungeteilt in eine der Tochterzellen hinein. Dadurch entstehen zwei Spermatozyten zweiter Ordnung, von denell der eine fünf, der andere sechs Chromosomen auf- 
weist. Bei der zweiten Reifungsteilung gehen dann aus den zwei Spermatozyten zweiter Ordnung vier Spermatiden hervor, von denen zwei je fünf und zwei je sechs Chromosomen besitzen. Während nun bei anderen Objekten die Chromosomen miteinander verklumpen, bleiben sie bei Ancyracanthus auch weiterhin einzeln nebeneinander liegen und sind noch an den fertigen Spermien zu zählen. Die Abbildungen, welche Mulsow gegeben hat, lassen es in der Tat gerechtfertigt erscheinen. die fünf bezw. sechs Chromatinkügelchen des Spermiums direkt als Chromosomen anzusprechen; an und für sich würde ich mehr dazu geneigt haben. sie als Partialkernchen aufzufassen.

Der Nebenke $\mathrm{n}$ n (Plastochondrienkörper) des Filariaspermiums stellt ein ziemlich voluminöses Gebilde dar, welches die ganze Breite des vorderen Spermienendes einnimmt. Er ist meistens entweder annibernd kugelig oder halbkugelig gestaltet; im letzteren Fall ist die konvexe seite nach voln gekehrt. Zuweilen ist er schalenförmig (Fig. 10). Andere Male hat er mehr die Form eines kurzen Zylinders, dessen Längsachse mit derjenigen der Zelle zusammenfällt (Fig. 9). An Präparaten, welche mit Flemmingschem Gemisch fixiert und mit Safranin gefürbt sind. ist er in bräunlichem Ton, etwas, aber nur wenig stärker als das Zytoplasmat, getärbt; im übrigen zeigt er ein homogenes Aussehen. Bei Anwendung der Eisenhilmatoxylinmethode (Fig. 6-10) nimmt er eine intensiv schwarye Färbung an. lässt aber bei genügendel Ausziehung Strukturverhältnisse erkennen; und zwar erscheint er entweder vakuolisiert oder grobbalkig (Fig. 6, 7 ) oder er weist, und zwar in sehr vielen Fällen, eine körnige oder körnig-fädige Beschaffenheit auf (Fig. \$).

In Fig. 39-48 habe ich schliesslich noch eine Anzahl Bilder zusammengestellt, wie man sie nach Fixierung mit SublimatAlkohol-Eisessig und Färbung mit Giemsalösung erhält. Sie sind untereinander recht verschieden. In einigen Präparaten treten in den Spermien nur die Chromatinkügelchen hervor, das ganze übrige Spermium dagegen ist gleichmässig rotviolett oder auch blauviolett gefärbt. In anderen Fallen dagegen zeigen sich verschiedene Teile des Spermiums verschieden tingiert; und zwar können abgesehen von den Chromatinkügelchen sowohl das Zytoplasma des Schwanzteils und der Nebenkern als auch die vor dem Nebenkern gelegene zytoplasmatische Kappe, welche die 
Mitwirkung der Plastosomen bei der Befruchtang des Eies.

Chromatinkügelchen einschliesst, eine besondere Fürbung aufweisen.

Da anzunehmen ist, dass die Filariaspermien mit denjenigen von Ancyracanthus nicht nur in bezug auf ihre Chromatinverhältnisse übereinstimmen, interessiert es, auch die übrige Schilderung zu vergleichen, welche $\mathrm{Muls}$ ow von den Ancyracanthusspermien gegeben hat. Mulsow, dessen Beobachtungen sich ausschliesslich auf das lebende Objekt und auf Totalpräparate der Geschlechtsorgane beziehen, welche aus den mit Sublimat-AlkoholEisessig fixierten Würmern unter der Lupe herauspräpariert, mit Boraxkarmin gefärbt, in Nelkenöl aufgehellt und entweder hierin oder in Kanadabalsam untersucht wurden, sagt, dass die fertigen Spermien von Ancyracanthus "Kugelform haben" und dass sie „zum grössten Teil aus einem Glanzkörper bestehen, dem eine kleine Kappe von körnigem Protoplasma aufgelagert ist. In diesem Protoplasma liegen die Chromosomen -." Bei den Filariaspermien muss ich die Existenz eines Glanzkörpers, wie ihn Mulsow bei Ancyracanthus beschreibt, in Abrede stellen. Der Glanzkörper von $\mathrm{Vulsow}$ bei Ancyracanthus entspricht wahrscheinlich dem von mir sogenannten zytoplasmatischen Schwanzteil des Filariaspermiums, die "Kappe von körnigem Protoplasma“ dagegen dem Nebenkern, von welchem $\mathrm{Mu}$ ls ow sonst gar nichts gesehen haben würde; die "Chromosomen" sind dann aber nicht, wie Mulsow schreibt, in dem „körnigen Protoplasma“, sondern vor ihm oder an der Seite desselben gelegen.

\section{Die unbefruchteten Eier und ihre Entwicklung.}

(Fig. 11-17.)

Der Ovarialschlauch von Filaria ist, verglichen mit demjenigen von Ascaris, verhältnismässig kurz und bietet daher eine günstige Gelegenheit, welche ich nicht unbenutzt gelassen liabe, um die Entwicklung der Eizellen zu studieren; jedoch habe ich mich dabei im wesentlichen auf eine Verfolgung ihrer plastosomatischen Strukturen beschränkt.

Das blinde Ende des Ovarialschlauchs ist dicht erfüllt von kleinen rundlichen Zellen, Oogonien, welche einen kugeligen Kern aufweisen, der durch den Besitz eines grossen Nukleolus ausgezeichnet ist. Das Zytoplasma schliesst zahlreiche, gewundene, durch Eisenhämatoxylin nach Fixierung mit modifiziertem 
Fl e m ming schem Gemisch schwarz färbbare Fäden, Plastokonten. ein, welche den Kern gleichmässig auf allen Seiten umgeben.

Indem die Eizellen in die Wachstumsperiode iibertreten, nehmen sie, grösser werdend, eine birnförmige Gestalt an (Fig. 11, 12). Benachbarte Eizellen hängen an den spitzen Enden, in welchen man häufig, besonders bei schon etwas stärker herangewachsenen Zellen, einen homogenen Körper erkennt (Fig. 13), untereinander zusammen. Die im Zytoplasma enthaltenen gewundenen Fäden wachsen gleichfalls und nehmen dabei nicht nur an Länge, sondern zunächst auch noch an Dicke zu; sehr bald aber (Fig. 13) wird eine Dickenabnahme an ilnen bemerkbar; die untere abgerundete Hälfte der birnförmigen zelle wird meistens vóllig von den Fïden erfüllt.

Noch grösser werdend streben die Eizellen einer ellipsoidischen Gestalt zu (Fig. 14, 15, 16). Die homogenen Kïrper, welche früher das zugespitzte Ende der Zelle einnahmen (Fig. 13). werden dabei, indem dieses sich mehr und mehr abrundet, ins Innere des Zytoplasmas aufgenommen (Fig. 15, 16). Van konstatiert nunmehr deutlich, dass sie sich ausserhalb der Zellen in einen Strang fortsetzen, welcher die gleiche Beschatfenheit wie sie selbst hat. Dieser Strang, eine sogenannte Rhachis, ist veristelt; die Enden der Ïste treten mit benachbarten Eizellen in Verbindung, indem sie sich ein Stück weit in die Zellen hineinerstrecken; die in Fig. 15 und 16 gezeichneten „homogenen Körper" sind selbst weiter nichts als solche Astenden.

Die Dickenabnahme der Plastokonten, welche schon auf dem Stadium der Fig. 13 bemerkbar war, setzt sich durch die folgenden Teile der Wachstumsperiode bis kurz vor ihrem Abschluss weiter fort. Die geschlängelten Plastokonten werden feiner, zugleich aber immer zahlreicher; dabei bestreben sie sich, eine ungefähr parallele Anordnung zur Längsachse der Zelle anzunebmen. Auf dem Stadium der Fig. 15 wird das Zytoplasma des Oozyten der Länge nach von zahlreichen feinen geschlangelten Faden dicht durchsetzt. In der Folge werden die Faden noch feiner und beginnen dann sich in Körnchen $z u$ zerlegen (Fig. 16). Wenn die Eier ihre definitive Grösse erreicht und sich von der Rhachis losgelöst haben (Fig. 17), sind sämtliche Fäden in kleine Körnchen zerfallen; zwischen den Körnchen sind Vakuolen in der Grundsubstanz des Zytoplasmas aufgetreten. 
Der Kern geht im Lauf der Wachstumsperiode aus der runden in eine ovale Form über, um schliesslich wieder zur runden zurückzukebren. Den Veränderungen, die sich in seinem Innern abspielen, habe ich keine weitere Beachtung geschenkt.

Ein in mancher Beziehung ähnliches Verhalten der Plastosomen, wie ich es bei der Entwicklung des Filariaeies beobachtet habe, ist schon 1891 von den Gebrüdern $Z 0 j a$ bei einem anderen Nematoden, nämlich beim Pferdespulwurm. mit Hilfe der Alt$\mathrm{m}$ an $\mathrm{n}$ schen Methode festgestellt worden.

Die „Plastidulen“, wie die beiden italienischen Autoren sagen, präsentieren sich bei Ascaris in den Zellen der Vermehrungsperiode als feine; mannigfach gewundene Fäden, welche das ganze Protoplasma erfüllen. In den Zellen der Wachstumszone haben sie die Gestalt ziemlich langer Fäden, die in mannigfacher Weise gewunden und verflochten, im allgemeinen jedoch parallel der Hauptachse der Zelle angeordnet sind; bei starker Vergrösserung lassen sie sich hïufig in Reihen sehr kleiner rundlicher Körner auflüsen. Die Fiaden sind zahlreicher in dem schmäleren, der Rhachis zugekehrten 'T'eil. wo sie zugleich länger sind. Dann finden sie sich, nicht sebr reichlich, zwischen den sphères hyalines und corpuscules réfringents, in weniger langen Reihen oder auch isoliert. Um den Kern herum sind sie leicht angehäuft: von ilım gehen Fiiden in unregelmässig radiärer Richtung aus. Eine etwas reichere Zone kommt auch unter der ganzen Zelloberfläche zur Beobachtung. In etwas weiter herangewachsenen Eiern sind die Fäden weniger lang, die zerstreuten Granula reichlicher und die Anhäufung gegen die Rhachis zu geringer. Wenn die Eier sich ablösen, bemerkt man immer noch an demjenigen Ende, nuit dem sie angeheftet waren, mehr zusammengruppierte Plastidulen, welche vorwiegend rund, nicht zu Fäden vereinigt und immer ziemlich klein sind.

In Eiern, welche die elliptische Form angenommen haben, finden sich lileine runde Plastidulen, ziemlich viel reichlicher um die "Polplatte" herum als im übrigen Ei; sie bilden hier häufig eine Anhäufung, von welcher strablige Reihen ausgehen können; im übrigen Ei liegen kleine runde Plastidulen mehr verstreut zwischen den geformten Elementen; an der Peripherie sind sie reichlicher.

Weitere Beobachtungen über die Plastosomen in den sich entwickelnden Oozyten von Ascaris stammen von Fräulein Schoonjans (1909), welcher die Zoja sche Abhandlung, die ich 1910 der Vergessenheit entrissen habe, unbekannt geblieben ist. Duesberg (1912, S. 716) und ich (1911,1, S. 702) haben die Angaben der Gebrüder Z oja bei gelegentlicher Nachprüfung bestätigt gefunden. Nachuntersuchungen am gleichen Objekt haben ferner Fauré-Fremiet (1913, S. 502) und Hirschler (1913, S. 375) vorgenommen. Letzterer findet in den Oozyten 
ausschliesslich Körner (Plastochondrien); augenscheinlich hat er ein Material bearbeitet, dessen Fixierung zu wünschen übrig liess.

Bei anderen Tieren als Nematoden sind Plastokonten in heranwachsenden Eizellen mehrfach beschrieben worden, z. B. von Bluntschli (1904) bei Ascidien (Cynthia), von Schaxel (1911) bei Echinodermen (Asterias und Holothuria), von Levi (1912) bei Amphibien (Geotriton). Tsukaguchi (1914) hat in grösseren Oozyten einer Meduse (Aurelia) "ziemlich lange Faden“ beobachtet, welche gegen Ende der Wachstumsperiode „Körnchen und kurzen Stäbchen" Platz machen. Nach der Literaturzusammenstellung, welche Duesberg (1912) gegeben hat, scheinen aber die Plastosomen der Eizellen a uch während der Wachstumsperiode gewöhnlich Körnerform zu besitzen.

\section{Das eben eingedrungene Spermium.}

(Fig. 18, 19 und $49-51$.)

Die Spermien erfüllen, wie gesagt, das oberste Uterusende in verschieden grosser Ausdehnung, vermögen aber auch, wie ich mehrfach konstatiert habe, in den Eileiter einzutreten. Spermien, welche im Eindringen in die Eizelle begriffen waren, habe ich nicht mit Sicherheit beobachtet. Möglicherweise erfolgt die Kopulation meistens schon im Eileiter.

Bei Fig. 18 und 19, denen Präparate zugrunde liegen, welche mit modifiziertem Flemmingschen Gemisch fixiert und mit Eisenhämatoxylin gefärbt worden sind, handelt es sich um befruchtete Eizellen, welche isoliert mitten in der das oberste Uterusende erfüllenden Spermienmasse gelegen waren. Solche Zellen zeigen eine von der ellipsoidischen mebr oder minder stark abweichende, mit buckelförmigen Vortreibungen besetzte, unregelmässige Gestalt, die wahrscheinlich der Ausdruck einer amöboiden Bewegung ist, welche die Eizellen zur Zeit der Befruchtung oder kurz nach Eintritt derselben ausführen. Die Spermien sind in Fig. 18 und 19 bereits völlig in die Eizellen aufgenommen, aber noch unmittelbar unter der Eiobertläche liegen geblieben; ihre Form ist länglich, der Längsdurchmesser der Eioberfläche parallel gelegen. Eine Eihaut scheint sich bereits gebildet und über dem eingedrungenen Spermium in Form einer Blase abgehoben zu haben. 
Die Eizelle beginnt alsbald zu einer annähernd ellipsoidischen Gestalt zurückzukehren; das Spermium bleibt zunächst noch unter der Zelloberflache liegen, geht aber aus der in die Länge gestreckten Form in eine mehr rundliche über. Es wird nunmehr in den meisten Fällen an einem Pol der Eizelle oder in der Nähe desselben gefunden; sein Eindringen muss also auch wohl hier erfolgt sein (Fig. 49-51, Fixierung mit SublimatAllohol-Eisessig, Giem s a farbung).

Von Bestandteilen des Spermiums erkennt man in den Figuren 18 und 19 den Nebenkern, umgeben von dem Plasmakörper des Spermiums, welcher sich seinerseits deutlich vom Eiplasma abgrenzt. Der plastosomatische Nebenkern bildet eine körnig-fädige Masse, welche durch Eisenhamatoxylin intensiv schwarz gefärbt ist. Dagegen erscheinen die kleinen Eiplastochondrien in Fig. 18 und 19 und ebenso in den folgenden Figuren nur noch in grauer Farbe, weil die zugrunde liegenden Priparate stärker differenziert sind als dasjenige, nach welchem Fig. 17 gezeichnet ist; die Folge davon ist. dass die grösseren Körner des Nebenkerns, welche die Schwarzfärbung energisch festhalten, um so deutlicher hervortreten.

Von den Chromatinkügelchen der Samenzelle ist in Fig. 18 und 19 (und auch in den folgenden Figuren 20-32 und in F'ig. 34) nichts wahrzunehmen. Während sie an den freien Spermien ausserordentlich leicht darzustellen sind, hat mir ihr Nachweis innerhalb der Eizelle während der ganzen Zeit, welche bis zur Bildung der ersten Richtungsspindel verläuft, anfangs grosse Schwierigkeiten bereitet. Van Beneden hat bereits 1883, S. 179 von den Ascarisspermien angegeben, dass ihre Kerne sich nach dem Eintritt der Befruchtung viel weniger intensiv färben lassen als vorher. Dass aber die Kernsubstanz des eingedrungenen Spermiums eine so starke Abneigung gegen Farbstoffe zeigt, wie man es bei Filaria beobachtet, hätte ich nicht für möglich gehalten.

Bei den mit Flemmingschem Gemisch fixierten Eiern habe ich die Chromatinkügelchen des aufgenommenen Spermiums nach Färbung mit Eisenhämatoxylin nur in ganz seltenen Fällen und auch dann fast immer nur zum Teil tingiert gefunden; Fürbungen mit Hämalaun oder mit Anilinfarbstoffen wie Safranin, Methylenblau u. a. blieben sogar völlig resultatlos. 
Ich ging dann zu dem Material über, welches ich mit Sublimat-Alkohol-Eisessig und mit Pikrinsäure-Formol-Eisessig behandelt hatte und färbte es zunächst mit Hämatoxylin und Karmin, dann mit Anilinfarbstoffen und Gemischen von solchen. wie Ehrlich-Biondischer Lösung und Methỵlgrün-Prronin nach Pappenheim. Auch hier war das Resultat mit Bezug auf die Chromatinkügelchen der eingedrungenen Samenzelle negativ: nur bei Anwendung der Methylgrüngemische nahmen sie in Spermien, welche ihre Lage unter der Zellobertlache bereits seit längerer Zeit aufgegeben hatten, einen leicht grünlichen T'on an.

Einige der angewandten Färbungen ergaben jedoch in anderer Hinsicht ein interessantes Resultat, insofern als sie zeigten, dass der Plasmakörper des aufgenommenen Spermiums mit gewissen basischen Anilinfarbstoffen wie Methylenblan nnd Pyronin stark färbbar geworden ist. Zum Beispiel \%eigten Präparate. die mit Flemmin $\mathrm{g}$ schem Gemisch fixiert und mit MethylenblanFuchsin $\mathrm{S}$ simultan gefärbt waren, die Chromatinkïgelchen der fr e i e n Spermien dunkelblau, die Plasmakörper der eingedrungenen heller, aber ebenfalls noch tiefblau geturbt. An Schnitten von Sublimat-Alkohol-Eisessig-Waterial, welche einer Loppelfärbung mit Methylgrün-I'yronin unterworfen waren, erschienen erstere schön grün. letztere intensiv rot tingiert. Die Chromatinkügelchen der eingedrungenen Spermien aber waren in beiden Fällen völlig ungefïrbt geblieben.

Erst als ich dann die Giemsafärbung auf die mit Sublimat-Alkohol-Eisessig fixierten Eier anwandte, gelang es mir ausser einer starken Blaufärbung des Plasmakörpers der aufgenommenen Spermien eine sehr schöne und intensive Rotfärbung ihrer Chromatinkügelchen zu erzielen. Die Blaufärbung des Plasmakörper's ist allerdings zunächst so kräftig (Fig. 49), dass dadurch die in ihm eingeschlossenen Chromatinkügelchen meistens verdeckt werden Sie beginnt aber gewöhnlich nach einigen Tagen zu verblassen und nunmehr treten die Chromatinkügelchen in dem eingedrungenen Spermium mit besonderer Deutlichkeit hervor (Fig. 50, วิ1).

Der blau gefärbte Plasmakörper erscheint übrigens bei der Giemsa färbung in der Regel nicht homogen, sondern undeutlich körnig. Diese Erscheinung möchte ich auf das Auftreten einer besonderen Granulation zurückführen, von welcher ich auch an 
Sublimat-Alkohol-Eisessig-Präparaten, die mit Eisenhämatoxylin tingiert worden waren, Andeutungen gesehen habe; es sind dies. Körner, welche mit denjenigen des Nebenkerns auf keinen Fall identisch sind.

Ausser den rot tingierten (hromatinkügelcben bleibt in dem abblassenden Spermienplasma noch ein kleiner rundlicher oder ovaler, stärker blau gefürbter Körper zurück (Fig. 50, 51), von welchem ich am freien Spermium nichts wahrgenommen habe; über seine Natur und seine weiteren Schicksale (er ist auch noch später. z. B. auf den Stadien der Figuren 53 und 55 , nachweisbar) vermag ich nichts auszusagen.

Das Eiprotoplasma erscheint an den Giemsapräparaten gleich nach ibrer Anfertigung blauviolett gefürbt, nimmt aber nach einiger Zeit einen rotvioletten Ton an; es zeigt einen undeutlich körnigen oder körnig-fädigen Bau, welchen ich in meinen Figuren nicht wiedergegeben babe.

VI. Die Veränderungen am befruchteten Ei bis zur Bildung der beiden Vorkerne. (Fig. 20-34 und 52-74.)

Bevor ich dazu übergehe, die weiteren Erscheinungen zu beschreiben, die sich an dem eingedrungenen Spermium abspielen, sei kurz erwähnt, dass der Eikern beim Herannahen der ersten Reifungsteilung die Vitte der Eizelle verlasst, sich an den einen, in meinen Figuren unteren Pol der'selben begibt und hier die beiden Richtungsteilungen durchmacht, bei welchen die Chromosomenzahl sechs beträgt. Ein Eingehen auf die Reifungsteilungen lag nicht im Plan meiner Arbeit.

Am entgegengesetzten, in meinen Figuren oberen Pol der Eizelle oder in seiner Nähe tritt kurz vor Beginn der ersten Reifungsteilung eine eigentümliche Bildung auf; es differenziert sich hier unter der Zelloberfläche ein grösserer heller Bezirk von annähernd ovaler form, welcher gegen das übrige Eizytoplasma deutlich abgegrenzt ist (Fig. 27, 28; über die scheinbar abweichende Lage des bellen Bezirks in Fig. 26 siehe Anm. auf folgender Seite). Im Bereich dieses hellen Bezirks liegt direkt an der Zellobertläche ein linsenförmiger Körper von homogenem Aussehen, welcher sich in der Folge unter Verkürzung seines. langen Durchmessers $z u$ einem mehr rundlichen Gebilde umwandelt; dieses löst sich vielfach, aber nicht immer, von der 
Zelloberfläche los und gerät mehr in das Innere des bellen Bezirks hinein (Fig. 30, 32-34); nicht selten sieht man blasse Fäden von ibm innerhalb des bellen Bezirks nach verschiedenen Richtungen ausgehen (besonders an Eiern, welche mit SublimatAlkohol-Eisessig fixiert sind). Über die Entstehung und Bedeutung dieser gesamten Bildung vermag ich nichts auszusagen, möchte aber glauben, dass sie ihrer Genese nach in Beziehung zu dem intrazellularen Teil des Rhachisstranges (Fig. 15, 16) zu bringen ist. Wo man, wie in den Fig. 29 und 31, nichts von ibr wahrnimmt, könnte sie etwas seitlich rom Zellpol gelegen haben und weggeschnitten sein; es ist aber auch möglich, dass sie nicht konstant vorhanden ist. Gegen Ende der ersten Richtungsteilung lückt sie von dem Pol, an welchem sie bisher gelegen war, weg an die Seite der Eizelle, ohne aber ihre Lage an der ZelloberHläche aufzugeben (Fig. 65-67). Auffallender Weise werden von diesem Zeitpunkt an häufig zwei (Fig. 66) oder sogar drei solcher Bildungen an verschiedenen Stellen unter der Zelloberfläche aufgefunden. Nach Ausstossung des $z$ we it e n Richtungskörpers ist in der Regel nichts mehr von ihnen wahrzunehmen.

Das eingedrungene Spermium haben wir zu einem Zeitpunkt verlassen, wo es als eine rundliche Masse an der Zelloberflache gelegen war. Bald darauf tremt es sich von der Zellobertläche und lagert sich in der Längsachse der annähernd ellipsoidischen Zelle auf der einen Seite des Eikerns. zwischen diesem und demjenigen Pol der Eizelle, welcher dem Richtungskörperpol entgegengesetzt ist. ${ }^{1}$ )

An Präparaten, welche mit modifiziertem Fl le m ming schen Gemisch fixiert und mit Eisenhämatoxylin gefürbt worden sind, erkennt man auf diesem Stadium (Fig. 20) den Nebenkern im Zentrum des Spermienplasmas als eine ungefähr kugelige Anhäufung intensiv schwarz tingierter Plastochondrien. Man sieht nun weiter, wie einzelne dieser Plastochondrien sich von der zentralen Ansamm-

I) Der dem Richtungskörperpol entgegengesetzte ist derselbe, an welchem der eben besprochene ${ }_{n}$ helle Bezirk ${ }^{"}$ auftritt. Das Spermium liegt also zwischen dem „bellen Bezirk ${ }^{*}$ und dem Kern. Eine Ausnahme bildet Fig. 26, in welcher der ${ }_{n}$ helle Bezirk" nnd das Spermium sich auf entgegengesetzten Seiten des Kerns finden. Diese Fig. 26 steht, verglichen mit den Figuren 20-25 und 27-36, wahrscheinlich auf dem Kopf: das Spermium hat ausnahmsweise seine Lage zwischen dem Richtungskörperpol und dem Kern genommen. 
Iung ablösen und aus dem Spermienkörper heraus in das Eizytoplasma übertreten, innerhalb dessen sie zunächst durch ihre Grösse deutlich erkennbar bleiben. Infolge der immer stärker werdenden Auswanderung wird die Körneranhäufung im Spermienkörper zusehends kleiner, während die Anzahl der im Eizytoplasma nachweisbaren grossen Plastochondrien immer mehr wächst (Fig. 21 fi.). In späterer Zeit (Fig. 25 ff.) nimmt man an den ausgewanderten Körnern vielfach Zerfallserscheinungen wahr; statt eines grösseren Korns findet man zwei oder drei oder vier kleinere. welche auf einem Haufen zusammenliegen.

In den Figuren 28-31 und besonders in Fig. 32 ist der Spermienkörper bis auf wenige Körner ausgeräumt. Die Zahl der im Eiprotoplasma sichtbaren grossen Plastochondrien hat aber nicht mehr zugenommen, in Fig. 32 sogar entschieden abgenommen. Der Grund könnte darin liegen, dass die Körner in der ganzen Eizelle verstreut sind und daher auf einem Schnitt nur zum Teil gesehen werden. Wahrscheinlicher ist mir aber, dass die meisten von ihnen schon in kleinere Körner zerlegt sind, welche sich von Eiplastochondrien nicht mehr unterscheiden lassen.

Nachdem sich die erste Richtungsspindel ausgebildet hat (Fig. 33, 34), ist der Zytoplasmakörper des Spermiums von Plastochondrien gänzlich frei geworden; auch im Eizytoplasma werden grössere Körner, welche man als noch unzerlegte männliche Plastochondrien ansprechen könnte, entweder nur vereinzelt (Fig. 33) oder überhaupt nicht mehr (Fig. 34) angetroffen. Zur Zeit der Ausstossung des ersten Richtungskörperchens ist das Zytoplasma der Eizelle jedenfalls ausschliesslich von kleinen Körnern durchsetzt, welche sämtlich das Kaliber der Eiplastochondrien besitzen.

Muss man nun annehmen, dass die männlichen Plastochondrien nach ihrer Zerlegung im Eizytoplasma resorbiert worden sind? Ein Blick auf die Plastosomen der Eizelle, besonders der heranwachsenden (Fig. 11-16) genügt, um zu erkennen, dass diese Strukturen offenbar eine hervorragende Wichtigkeit besitzen; schon deshalb ist es wenig wahrscheinlich, dass die entsprechenden Strukturen des Spermiums dem Untergang bestimmt sein sollten. Wir wissen ferner, dass die Plastosomen mit den Faiden Flemmings von 1882 und den Granulis von Altmann (1890) identisch sind (Meves 1908, 1, 1910, 1914. 3, 
1915), dass sie also ganz ursprüngliche '/yytoplasmabestandteile darstellen. Wenn solche durch das Spermium in das Ei hineintransportiert werden, so erscheint mir ausgeschlossen, dass sie dort spurlos verschwinden sollten. Zugunsten der letzteren Annahme lässt sich kaum etwas anderes geltend machen, als dass eine Persistenz der mämnlichen Plastochondrien im Ei mit der Uonopolstellung unvereinbar ist, welche dem Chromatin der Samenzelle noch voll vielen Seiten bei der Übertragung erblicher Eigenschaften eingerüumt wird.

Vergleicht man die Aussaat männlicher I'lastochondrien, wie sie sich bei Filaria abspielt, mit dem gleichen Vorgang bei Ascaris (M ves 1911, 1), so ergeben sich folgende ['nterschiede: Bei Ascaris wandern die männlichen Plastochondrien aus dem Spermienkörper erst aus, nachdem sie sich zerlegt baben; bei Filaria dagegen treten sie unzerlegt in das Eizytoplasma über und zerfallen erst hinterher. Das Spermium wird ferner im Ei des I'ferdespulwurms zur Zeit, wo die männlichen Plastochondrien ausgesït werden, dicht von Eiplastochondrien umhüllt, welche sich von allen Seiten her im Umkreis desselben ansammeln: im Filariaei dagegen lassen die weiblichen I'lastochondrien keine Lageveränderungen erkennen.

Der aus zytoplasmatischer "(rundsubstanz" bestehende Körper der Samenzelle wird im Filariaei besonders, nachdem die erste Reifungsteilung begonnen hat, ${ }^{1}$ ) immer kleiner. Diese Verkleinerung ist zum Teil auf die Auswanderung der männlichen Plastochondrien zurückzufüluren; daneben tritt aber eine wirkliche Abnahme der Grundsubstanz, wahrscheinlich durch Resorption, ein. An Präparaten, welche mit modifiziertem Flemmingschen Gemisch fixiert und mit Eisenhämatoxylin gefärbt worden sind, kann man zu der Meinung kommen, dass der l'lasmakörper des Spermiums nach Ablauf der ersten Reifungsteilung völlig geschwunden ist (Fig. 65 ff.). Gie msa farbungen der mit SublimatAlkohol-Eisessig fixierten Eier zeigen aber, dass 'Teile davon noch längere Zeit persistieren können; man vergleiche Fig. 62, wo ein strangfölmiger Rest am oberen Rand des männlichen Vorkerns erhalten ist. Wie ich schon früher mit Bezug auf das Ascarisspermium bemerkt habe (1911, 1, S. 709), lässt sich die Möglichkeit nicht

${ }^{1)}$ In den Prophasen derselben ist er häufig vorübergehend kugelschalenförmig umgestaltet (vergl. Fig. 59). 
ausschliessen, dass von der Grundmasse des Spermienzytoplasmas Wirkungen irgendwelcher Art auf das Ei ausgehen.

Als eine eigentümliche Erscheinung am Spermienzytoplasma ist noch das Auftreten einer hellen Vakuole zu erwähnen, welche gewöhnlich am Rande desselben gelegen und von einer Membran eingeschlossen ist (Fig. 23, 25-27, 29, 31); die letztere ist in der Regel mit münnlichen Plastochondrien besetzt. Wenn die erste Richtungsspindel sich ihrer Fertigstellung nilhert, scheint die Takuole sich vom Zytoplasma der Samenzelle loszulösen und, indem ihr bisher heller Inhalt sich trübt, in ein homogen aussehendes Kügelchen überzugehen, wie es z. B. in Fig. 33 oberhalb des (die Chromatinkügelchen einschliessenden) Zirtoplasmarestes des Spermiums gelegen ist. Dieses Kügelchen kann noch längere Zeit persistieren. nicht nur wïhrend der Reifungsteilungen (Fig. 65, 69), sondern auch noch auf dem Stadium der Vorkerne (Fig. 70-72) und sogar noch im Verlauf der ersten Furchungsteilung.

Es erübrigt schliesslich, die Chromatinkügelchen des Spermiums in ihrem weiteren Verhalten zu verfolgen. In der eben eingedrungenen Samenzelle vermochte ich sie, wie gesagt, nur durch Giemsafü $\mathrm{rbu} n g$ an meinem Sublimat-AlkoholEisessig-Material darzustellen und glückte mir eine scharfe Färbung derselben auch in der spateren Zeit bis zum Auftreten der ersten Richtungsspindel ausschliesslich auf diese Weise.

Wie die Giemsapräparate zeigen, nehmen die Chromatinkügelchen des Spermiums nach dem Eindringen desselben allmählich an Grösse zu (Fig. ว2 fir.). Dabei bleiben sie auch weiterhin völlig voneinander isoliert, wenn sie auch mitunter so eng zusammen liegen, dass sie untereinander verbacken erscheinen. Zuweilen liegen sie sümtlich oder zum Teil ausserhalb des Spermienkörpers im Eizytoplasma; hier vergrössern sie sich schneller, als wenn sie im Spermienkörper eingeschlossen bleiben (Fig. 533).

Nachdem die erste Richtungsspindel die Höhe ihrer Ausbildung erreicht hat, kann man die Chromatinkügelchen des Spermiums auch bei anderer als Giemsafärbung wahrnehmen; so z. B. in Priparaten, welche mit modifiziertem Fle $\mathrm{mm}$ in $\mathrm{g}$ schen Gemisch tixiert und mit Eisenbämatoxylin tingiert sind; in diesen erscheinen sie allerdings zunächst gewöhnlich nur in ganz blassem 
Ton (Fig. 63, 64). Erst nach Ausstossung des ersten Richtungskörpers werden sie auch auf die eben genannte Weise stärker färbbar (Fig. 65) und beginnen nunmehr Formveränderungen zu zeigen (Fig. 66); jedes einzelne zerfallt in zwei oder mehr bleinere Körner. Weiter bilden sie sich zu einem Samenkern um, der zuniichst vielfach sehr unregelmässig gestaltet ist (Fig. 67, 69) und erst allmählich Bläschenform annimmt (Fig. 72, 73). Haufig aber liegen die fünf oder sechs Chromatinkügelchen, welche den Samenkern bilden sollen, nicht sümtlich miteinander vereinigt, sondern sind auf zwei oder auch drei Häufchen rereinigt, welche durch Zwischenräume voneinander getrennt sind (Fig. 68). Dann entsteht nicht ein einheitlicher Samenkern, sondern deren zwei (Fig. 70, 71) oder drei, welche heranwachsen und bis zur ersten Furchungsteilung isoliert erhalten bleiben (Fig. 74). Die Bildung des männlichen Vorkerns beginnt gewöhnlich etwas früher als die des weiblichen. In dem ersteren ist bald gar keine chromatische Substanz mehr zu erkennen; dagegen bleiben in dem weiblichen Kern noch längere \%eit Chromatinklumpen sichtbar, deren Anzahl derjenigen der Chromosomen entspricht (lig. 62. 72 ).

Bei Ancyracanthus hat Mulsow von einer Abneigung der Chromatinkügelchen des eingedrungenen Spermiums gegen Farbstoffe nichts erwähnt. Bei einer Betrachtung seiner Figuren komme ich jedoch zu dem Ergebnis, dass die Sache hier wahrscheinlich ebenso wie bei Filaria liegt. Mulsow bildet zwei Spermien ab, welche nach ihm im Eindringen begriften sind, ron denen das eine fünf, das andere sechs Chromatinkiügelchen zeigt: dann aber führt er das männliche Chromatin innerhalb des Eies erst wieder vor, nachdem es sich zu einem grossen Samenkern umgebildet hat. Im Text heisst es: „Das (eingedrungene) Spermatozoon bleibt zunächst untätig im Protoplasma des Eies an einer beliebigen Stelle liegen. Während dieser Ruhe verklumpen die Chromosomen meistens miteinander, so dass ihre Zahl dann nicht mehr festzustellen ist." Hierzu ist zu bemerken, dass die Stelle, wo das Spermium sich lagert, im Filariaei wenigstens durchaus keine beliebige ist; auch kann von einer meistens erfolgenden Verklumpung der männlichen Chromatinkügelchen bei diesem Wurm nicht die Rede sein. 
Mitwirkang der Plastosomen bei der Befruchtung des Eies.

VII. Der Beginn der Furchung. (Fig. $35-38$ und $75-77$.)

Mit dem Herannahen der ersten Furchungsteilung treten in den beiden Vorkernen wieder Chromosomen auf (Fig. 75). Die Eizelle der Fig. 35 zeigt die erste Furchungsspindel auf der Höhe der Ausbildung. Hat man die in der Äquatorialebene der Spindel versammelten Chromosomen in Polansicht vor sich, so kann man feststellen, dass ihre Zahl bald elf (Fig. 76), bald zwölf (Fig. 7i) betrigt. Nach den Ergebnissen neuerer Forschung würde sich die Eizelle im ersteren Fall zu einem Kännchen, im letzteren zu einem Weibchen entwickelt haben.

Im Zytoplasma ist in Fig. 35 gegen das zuletzt besprochene Stadium keine Veründerung eingetreten. Die Plastochondrien sind in der ganzen Zelle gleichmässig verteilt geblieben; von einer Anhäufung um die Zentrosomen, wie man sie im Ascarisei beobachtet, ist keine Spur wahrzunehmen. ${ }^{1}$ )

Auf dem Zweizellenstadium der Fig. 36 sind die Valiuolen im Zýtoplasma, von welchen übrigens an manchen Präparatenserien auch vorher wenig oder gar nichts zu erkennen ist, völlig geschwunden. Dagegen sind eine Anzahl homogen aussehender Ballen sichtbal geworden, welche sich mit Eisenhämatoxylin schwarz färben lassen, den Farbstoff aber bei der Differenzierung ziemlich leicht wieder abgeben. Mitunter trifft man solche Ballen bereits in den Prophasen der ersten Furchungsteilung an; sie scheinen auf den späteren Stadien der Teilung wieder zu verschwinden, um dann nach Ablauf derselben von neuem aufzutreten.

Auf dem Stadium der Fig. 37, in welcher acht Blastomeren auf dem Schnitt getroffen sind, ist der Bau des Zytoplasmas der gleiche wie in Fig. 36. Gehen wir dagegen zu dem in

1) In einer früheren Arbeit $(1914,1)$ habe ich S. 107 gesagt, dass Anlaäufungen von Plastochondrien in der nächsten Umgebung der Zentrosomen, wie wir sie im Ei und in den Blastomeren von Ascaris antreffen, uns bei anderen Tieren bisher nicht mit Sicherheit bekannt seien. Dieser Satz bedarf der Berichtigung insofern, als $\mathrm{Lams} 1910 \mathrm{im} \mathrm{Ei}$ von Arion empiricorum im Umkreis der Zentrosomen analoge Plastochondrienanhäufungen beschrieben hat, welche hier zuweilen sehr regelmässig in Form zweier, durch eine homogene Zone getrennter Kugelschalen angeordnet sind (H. L a ms, Recherches sur l'oeuf d'Arion empiricorum. (Accroissement, maturation, fécondation, segmentation). Mémoires in $-4^{\circ}$ publiés par la Classe des Sciences de l'Académie Royale de Belgique, t. II, 1910.) 
Fig. 38 abgebildeten, bereits weit vorgeschrittenen Furchungsstadium über, so ist nunmehr das durch die Anwesenheit zahlreicher kleiner Plastochondrien bedingte feinkörnige Aussehen des Zytoplasmas völlig verschwunden; auch von den eben beschriebenen Ballen ist nichts mehr wabrzunehmen. Das Zytoplasma der Furchungszellen bietet vielmehr auf diesem Stadium folgendes Bild: es besteht aus einer homogen aussehenden Grundsubstanz, in welcher einzelne dicke Plastokonten eingebettet sind.

Die Herausbildung dieser dicken Plastokonten habe ich nicht verfolgt: wie sie aber auch vor sich gegangen sein möge, die Annalıme erscheint mir unabweisbar, dass nicht nur die Eiplastochondrien, sondern auch die in der Eizelle ausgesiten und zerlegten männlichen Plastochondrien an der Entstehung der Plastokonten Anteil genommen haben. Minnliche und weibliche Plastochondrien müssen sich also zu einem Y ischprodukt vereinigt haben. Dadurch erfüllen sie eine Eorderung, welche $\mathrm{Na}$ ageli (1884) an die elterlichen Idioplasmakörper stellt: dass sie sich vereinigen, um ein neues Idioplasma, dasjenige des Kindes, zu bilden.

Naegeli, welcher das Idioplasma aus Strüngen bestehen lässt, die sich ihrelseits aus parallelen Reihen von Micellen zusammensetzen, hat das Zustandekommen der Vereinigung zwischen männlichen und weiblichen Idioplasmakörpern rom theoretischen Standpunkt in eingehendster Weise erörtert. Er nimmt an, dass sie sich gegenseitig in derselben Weise wie Eizelle und Spermium anziehen und sich infolge davon aneinanderlegen. Weiter lösen sich entweder Micellen von dem einen idioplasmatischen System nach und nach $a b$ und wandern in das andere hinüber; oder aber münnliche und weibliche Idioplasmakörper bleiben intakt und "wirken bloss gegenseitig auf das Wachstum der einen und anderen so ein, dass dasselbe $z u$ einer mittleren Bildung hinstrebt".

Auch de Vries (1889) und O. Hertwig (1890) sind durch ihre Betrachtungen zu dem Ergebnis geführt worden, dass bei der Befruchtung eine "Durchdringung und Vermischung" der Erbanlagen stattfindet.

An meinen Prïparaten von Ascaris habe ich 1911, 1 beobachtet, dass die im $\mathrm{Ei}$ vorhandenen Plastochondrien nach 
Beendigung der ersten Reifungsteilung deutlich vergrössert erschienen, und habe tür möglich erklärt, dass diese Vergrösserung mit einer Kopulation zwischen männlichen und weiblichen Plastochondrien zusammenhängen könne, habe aber allerdings bemerken müssen. dass sie vielleicht auch auf Rechnung einer Quellung zu setzen sei, welche eingetreten sein könnte, weil. das fixierende Reagens die auf diesen Stadien bereits stark verdiclite Dotterhaut erst nach Ablauf einiger Zeit $z u$ durchdringen vermag. Bei Filaria vollzieht sich die erste Vereinigung zwischen männlichen und weiblichen Plastochondrien möglicherweise erst im Lauf der Furchung. Bei Phallusia kann es nach meiner Darstellung (1913) jedenfalls nicht anders sein, da in der ungefurchten Eizelle die weiblichen Plastochondrien der Menge nach enorm überwiegen und vor Eintritt einer Vereinigung mit den entsprechenden männlichen Gebilden erst die vorhandene Ungleichheit durch Wachstum der letzteren beseitigt werden muss.

Das, was sich demnach für das Zytoplasma als notwendige Annahme ergibt, dass sich eine Vereinigung seiner elterlichen Erbanlagen verschieden lange nach der Kopulation von Ei- und Samenzelle bis in die Zeit der Furchung hinein verschieben kann, steht mit Bezug auf den Kern, welcher nach Pfeffer (1897, S. 43) mit dem Zytoplasma in Symbiose ${ }^{1}$ ) lebt. schon lange fest, wenigstens für jeden, der nicht Anhänger der Chromosomenindividualität ist. Denn, während im Seeigelei Ei- und Samenkern kurze Zeit nach dem Eindringen des Spermiums miteinander verschmelzen (O. Hertwig, 1875), kann nach den Beobachtungen Van Benedens (1883) im Ei des Pferdespulwurms eine Vermischung des väterlichen und mütterlichen Chromatins ja frühestens nach Ablauf der ersten Furchungsteilung stattinden; im befruchteten Copepodenei bleiben die elterlichen Idioplasmakörper des Kerns sogar wälrend der ganzen ersten Entwicklung getrennt (Rückert, 1895 und $\mathrm{H}$ a ecker, 1896).

Ieine zuerst 1908 ausgesprochene Forderung, dass die männlichen und weiblichen Plastochondrien sich bei der Be-

1) Diese Anschauung wird auffallenderweise auch von einem der überzeugtesten Anhänger der $\mathrm{O}$. Hertwig-Strasburgerschen Vererbungslehre, nämlich von Boveri (1904, S. 90), akzeptiert; auffallenderweise: denn die beiden in Symbiose lebenden Organismen vererben doch jeder seine Eigenschaften selbständig und nicht der eine diejenigen des anderen mit. 
fruchtung vereinigen müssen, ist mehrfach auf Ablehnung gestossen. Demgegenüber möchte ich hier noch zunächst darauf hinweisen, dass auch Strasburger 1877 (S. 509) vorübergehend gegenüber 0 . Hertwig den Satz verteidigt hat, dass nicht bloss die Zellkerne, sondern überhaupt die gleichwertigen Teile der kopulierenden Zellen sich im Geschlechtsakt vereinigen und dass hierin das Wesen der Befruchtung bestehe. Von besonderem Interesse abel war mir der folgende literarische Fiund, welchen ich kürzlich gemacht habe. Delage hat in seinem $1895 \mathrm{er}-$ schienenen Werke L'hérédité bei einer Besprechung der Altmannschen Granulalehre geprüft, was Altmann selbst unterlassen hatte, wie die .Bioblasten ${ }^{*}$ sich zu dem Vererbungsproblem stellen, und dabei schon damals eine Vereinigung zwischen väterlichen and mütterlichen Körnern gefordert; er schreibt S. 503 folgendes:

"Altmannn aprìs ctre arrivé a cette conclusion que ses bioblastes sont les facteurs des propriétés de l'organisme, sarrête brusquement sans chercher a voir si des facteurs ainsi constitues permettent dexpliquer les phénomènes biologiques. Il se contente de présenter sous une forme concrète les unités hypothétiques des autres auteurs; de dire à Spencer à Haeckel, a Darwin, à Naegeli, à deVries, ì Hertwig, ì Wiesner, etc.: Voila vos unites physiologiques, vos plastidules, vos gemmules, vos micelles, vos pangenes, ros idioblastes, vos plasomes, etc.; ils ne sont point ce que vous avez imagine, ce ne sont que de petits appareils doués de propriétés chimiques définies. - Cela est fort bien, mais il faudrait montrer qu' ainsi constitués ils conservent les propriétés grâce auxquelles ces particules hypothétiques expliquaient plus ou moins les phénomènes de la vie. Altmann ne saurait prétendre avoir si rigoureusement démontré que les granules sont les facteurs des propriétés organiques qu'il soit dispensé de s'inquiéter des conséquences de sa conclusion. Il devait donc montrer comment ses bioblastes s'accommoderaient avec les problèmes de l'hérédité, de l'ontogénèse, de la variation, de l'adaption, etc. Il s'est burné à tracer quelques linéaments de la phylogénèse primitive. Ce n'est point assez, car il y a dans l'application des bioblastes à certains problèmes des difficultés très graves."

In einer Anmerkung zu letzterem Satz heisst es:

"Le nombre de leurs variétés doit être très considérable dans un organisme compliqué. Leur taille cependant n'est jamais très petite puisqu'elle reste toujours dans les limites de la visibilité.

On comprendrait à la rigueur que le nombre nécessaire puisse trouver place dans l'œuf. Mais dans le spermatozoïde, cette difficulté se complique d'une autre. C'est surtout, on peut dire c'est exclusivement, dans le cytoplasma que lion trouve des granules. Ceux du noyau sont fortement douteux 
et 1 ltmann lui-même en parle avec beaucoup moins d'assurance que de ceux du corps cellulaire. Or le spermatozoïde est presque entièrement formé de substance nucléaire. La portion cytoplasmique, que peut-être il renferme en lui, est de volume si minime qu'elle ne pourrait donner asile qu'a des particules de taille extrêmement inférieure à celle des granules, partant invisibles, et par suite lypothétiques, ce qui leur ôte le principal mérite des granules.

Mais aumettons que les bioblastes ultramicroscopiques, admis par une induction fondée sur les bioblastes visibles, puissent donner au spermatozoïde les propriétés nécessaires. Allmettons que ces bioblastes spermatiques ultramicroscopiques grussissent ensuite dans l'œuf fécondé et deviennent des granules ordinaires.

Le protoplasma de l'embryon contiendra donc deux bioblastes de chante espèce, un paternel et un maternel, qui pourraient, it la rigueur, expliquer la forme mixte des caractères exprimés. Mais il est évident que le nombre des bioblastes me saurait doubler ainsi ì chaque génération et qu'un phénomène de réduction doit se produire sous une forme quelquonque. La division réductrice ne peut l'expliquer, car elle ne pourrait qu'éliminer une moitic des bioblastes paternels et maternels, et il arriverait certainement que ceux de la même sorte se trouveraient souvent expulsés des deux côtés a la fois et manqueruient dans le produit. On ne peut qu ${ }^{*}$ imaginer, après la fécondation. une fusinn de deux bioblastes en un. ${ }^{1}$ ) Or Altmann n'a jamais signalé de phénomène de ce genre et s'il l'admettait ce ne pourrait itre qu'llypothétiquement. L'idée qu'il se fait de la nature des bioblastes n'est pas conciliable avec cette hypothèse. Deux sphórules formées senlement de sulustance chimique peuvent se fusionner lursqu'elles sont petites et grossir ensuite seulement autant qu'eût fait une seule. Mais les bioblastes sont. d'après lui, des sortes de cristaux organiques, en tout cas des agregats doués d'une structure qui intervient dans leurs propriétés. En ce cas, ils ne peuvent que se juxtaposer, et, au bout d'un nombre suffisant de générations, il n'y a plus place pour le grand nombre qui doit se trouver dans un seul granule...

Admettons yu'Altmin n ou quelque autre soit en état de répondre a toutes ces objections, il est évident qu'il ne saurait le faire sans faire intervenir des hypothèses et c'est là seulement ce que nous voulons démontrer pour le moment."

Ich möchte zu diesen Ausführungen bemerken, dass die Bioblasten des Spelmiums ja durchaus nicht, wie Del age damals (1895) annahm, ultramikroskopisch sind. Ich Lalte es ferner nicht für nötig, mit Delage die Existenz verschiedener Arten von Bioblasten anzunehmen. Die Hypothesen, deren wir für unsere Anschauung bedürten, nach welcher die Bioblasten (Plastochondrien bezw. Plastosomen) Vererbungsträger darstellen, werden uns teil-

1) Von mir gesperit. 
weise durch die $\mathrm{Na}$ ge $\mathrm{g}$ ische Idioplasmatheorie an die Hand gegeben (vergl. Meves, 1908, 1).

In den ersten Blastomeren sich furchender Eier hat man bishel, so viel ich weiss, stets (ebenso wie bei Filaria) Körner, Plastochondrien, gefunden; in den Embryonalzellen dagegen sind jedenfalls bei Wirbeltieren Fiaden vorhanden. Bei Amphibien (Triton) wurde eine Umwandlung der Körner in ausserordentlicl dünne schlanke Füden von D uesberg (1910.2) schon in den Zellen der Gastrula beobachtet. Beim Kaninchen dagegen erfolgt, ebenfalls nach Duesberg $(1910,1)$, die Bildung von Füden verhältnismässig spät, am vierten bis fünften Tage der Entwicklung: noch später nach Rubaschkin (1910) beim Meerschweinchen: während Levi neuerdings (1914) bei der Fledermaus konstatiert hat, dass die Plastochondrien noch im Laufe der Furchung (rom 22-30-Zellenstadium an) in Plastokonten übergehen. Levi erklärt, dass, die Verschiedenbeiten in der Form der Chondriosomen in den frühen Stadien der Entwicklung . . . . . sicher vongrosser Bedeutung sind."

\section{Schluss.}

Die vorliegende Untersuchung bildet eine weitere Stütze für meine Anschauung, nach weIcher die plastosomatischen Bestandteile des Spermiums bei der Übertragung der erblichen Eigenschaften beteiligt sind.

Der Umstand, dass eine Auswanderung ron Plastosomen aus dem Spermium in das Eizytoplasma sich bereits innerhalb verhaltnismässig kurzer Zeit in zwei Fillen, bei Ascaris und Filaria, direkt hat nachweisen lassen, bestärkt mich in der Überzeugung, dass die Befunde am Säugetier- und Seeigelei, welche meiner eben genannten Auffassung auf den ersten Blick $\% u$ widersprechen scheinen, sich ihr in der schon früher vermuteten Weise ebenfalls fügen werden.

Bei Säugetieren ist die von Van der Stricht (1909) und Lams (1910) entdeckte Tatsache, dass der Spermienschwanz, dessen Mittel- oder Verbindungsstück mit einer Plastochondrienhülle versehen ist, in die eine der beiden ersten Blastomeren übergeht, kürzlich auch von Levi (1914) bestätigt worden. Van der Stricht, Lams und Henneguy (Diskussion zu dem Fortrag von Lams, 1910) hatten an diesen Befund die Hypothese geknüpft, welcher auch Levi (1914) nicht widerspricht, dass diejenige Blastomere, welche den Spermienschwanz erhäl, den eigentlichen Embryo, die andere den sogenannten Trophoblasten (im Sinne von Hubrecht) bildet. Sobott, a hat diesen Schluss 
zunächst (1913,1, S. 16) als "sehr voreilig" bezeichnet, hat aber noch im selben Jahre $(1913,2)$ seinerseits ebenfalls eine Cngleichwertigkeit der beiden ersten Blastomeren angenommen, in demselben Sinne, dass die eine Blastomere den Embryo, die andere den Trophoblasten oder das "ausserembryonale Material“ bildet, um eine Hypothese über die Entstehung eineiiger Zwillinge des Menschen und der Polyembryonie bei den Gürteltieren darauf aufzubauen.

Beim Seeigelei habe ich kürzlich (1914.2) zeigen können, dass das plastosomatische Mittelstück des Samenfadens, welches bei der ersten Furchungsteilung in eine der beiden Blastomeren übergeht (Meves, 1912), auch im weiteren Verlauf der Furchung: erhalten bleibt; ich habe in einer grossen Anzahl von Keimen verschiedenen Alters bis zum 32-Zellenstadium inkl. Wittelstuicke aufgefunden, welche in ihrer Form völlig unveriandert waren. Die Vermutung, welche ich im Anschluss an diese Beobachtungen ausgesprochen habe, basiert auf der latsache, dass der junge Seeigel aus dem sogenannten Pluteus nicht direkt oder durch weitere Umwandlung, sondern als ein Neugebilde aus einer Ektodermeinstülpung, der sogenannten seeigelanlage oder seeigelscheibe, entsteht, wobei zablreiche Teile des Larvenkörpers, welche zu dem neuen Bau nicht benutzt werden, zu Grunde gehen. Und zwar glaube ich annehmen $z u$ dürfen, dass die Substanz des Mittelstücks in die Zellen der "Seeigelanlage“ übergeht, aus welcher sich, soviel ich aus der Literatur zu entnehmen vermag, sämtliche oder fast sämtliche Teile des jungen Seeigels mit Ausnahme des Darms (oder eines 'Teils desselben) und der Vasoperitonealblasen bilden. Die Zellen der zuletzt genannten Organe würden demnach allerdings keine münnlichen Plastosomen erhalten; die Möglichkeit aber, lass fast der ganze übrige Leib des jungen Seeigels durch das Mittelstück des Samenfadens väterliche Eigenschaften ererbt, bleibt bestehen. Die spätere Metamorphose des Seeigels ist übrigens ganz ausserordentlich kompliziert und trotz verschiedener auf diesen Punkt gerichteter ausgezeichneter Untersuchungen noch keineswegs genügend aufgeklärt.

Die Tatsache, welche sich demnach aus den Befunden am Säugetier- und Seeigelei zu ergeben scheint, dass die männliche plastosomatische Substanz nicht an vergängliche embryonale 
Bildungen verschwendet, sondern für das definitive Tier aufgespart wird, würde nur geeignet sein, unsere Wertschätzung der Plastosomen in ihrer Eigenschaft als Vererbungsträger zu steigern.

\section{Literaturverzeichnis.}

Altmann, R., 1890: Die Elementarorganismen und ihre Beziehungen zu den Zellen.

Bluntschli, H., 1904: Beobachtungen am Ovarialei der Monascidie Cynthia microcosmus. Norph. Jahrb., Bd. 32.

Boveri, Th., 1904: Ergebnisse über die Konstitution der chromatischen Substanz des Zellkerns. Jena.

Delage, Y., 1895: La structure du protoplasma et les théories sur l'hérédité. Paris.

Della Valle, P., 1909: Ljorganizzazione della cromatina studiata mediante il numero dei cromosomi. Archivio Zoologico, vol. 4.

De Vries, H., 1889: Intrazellulare Pangenesis. Jena.

Duesberg, J., 1910, 1: Sur la continuité des éléments mitochondriaux des cellules sexuelles et des chondriosomes des cellules embryonnaires. Anat. Anz., Bd. 35 .

Derselbe. 1910, 2: Nouvelles recherches sur l'appareil mitochondrial des cellules séminales. Arch. f. Zellforschung, Bd. 6 .

Derselbe, 1912: Plastosomen, ,apparato reticulare interno" und Chromidialapparat. Ergebn. d. Anat. u. Entwicklungsgesch., Bd. 20.

Fauré-Fremiet, E., 1913: Le cycle germinatif chez l'Ascaris megalocephala. Arch. d'anat. micr., t. 15.

Fick. R., 1905: Betrachtungen über die Chromosomen, ihre Individualität, Reduktion und Vererbung. Arch. f. Anat. u. Physiol., Anat. Abt., Suppl.

Derselbe, 1907: Vererbungstragen, Reduktions- und Chromosomenhypothesen, Bastardregeln. Ergebn. d. Anat. u. Entwickinngsgesch., Bd. 16, 1906.

Derselbe, 1908: Zur Konjugation der Chromosomen. Arch. f. Zellforsch., Bd. 1.

Flemming, W., 1882: Zellsubstanz, Kern und Zellteilung.

Ha ecker, V., 1896: Über die Selbständigkeit der väterlichen und mütterlichen Kernbestandteile während der Embryonalentwicklung von Cyclops. trch. f. mikr. Anat. u. Entwicklungsgesch., Bd. 46.

Held, H., 1912: Über den Vorgang der Befruchtung bei Ascaris megalocephala. Ber. d. Kgl. sächs. Ges. d. Wiss., math.-phys. IKl, und Verhandl. der Anat. Ges. auf d. 26. Vers. in München.

Hertwig, 0., 1875: Beiträge zur Kenntnis der Bildung, Befruchtung und Teilung des tierischen Eies. Morphol. Jahrbuch, Bd. 1.

Derselbe, 1890: Vergleich der Ei- und Samenbildung bei den Nematoden. Eine Grundlage für zelluläre Streitfragen. Arch. f. mikr. Anat., Bd. 36 . 
Hirschler, J., 1913: Über die Plasmastrukturen (Mitochondrien, Golgischer Apparat $u$. a.) in den Geschlechtszellen der Ascariden. Arch. f. Zellforschung, Bd. 9.

Jost, L., 1904: Vorlesungen über Pflanzenphysiologie. Jena.

La ms, H., 1910: Recherches sur l'œuf de Cobaye (Cavia Cobaya). Maturation, Fécondation, Segmentation. Comptes rendus de l'Association des Anatomistes, douzième Réunion, Bruxelles.

Levi, G.. 1912: I condriosomi nell' oocite degli Anfibi. Monitore zool. italiano, anno 23.

Derselbe, 1913: Note citologiche sulle cellule somatiche dell' ovaio dei Mammiferi. Arch. f. Zellforschung. Bd. 11.

Derselbe. 1914: Das Verhalten der Chondriosomen bei den frühesten Entwicklungsstadien der Säugetiere. Verh. d. Anat. Gesellsch. auf der 28. Vers. in Innsbruck.

Meves, Fr., 1900: Über den von v. la Valette St. George entdeckten Nebenkern (Mitochondrienkörper) der Samenzellen. Arch. f. mikr. Anat., Bd. 56 .

Derselbe, 190:3: Über oligopyrene und apyrene Spermien und über ihre Entstehung, nach Beobaclitungen an Paludina und Pygaera. Arch. $f$. milir. Anat., Bd. 61.

Derselbe. 1907: Die Spermatozytenteilungen bei der Honigbiene (Apis mellifica L.), nebst Bemerkungen äber Chromatinredulktion. trch. f. mikr. Anat., Bd. 70 .

Derselbe, 1908, 1: Die Chondriosomen als Träger erblicher Anlagen. Cytologische Studien am Hühnerembryo. Arch. f. mikr. Anat., Bd. 72.

Derselbe, 1908, 2: Es gibt keine parallele Konjugation der Chromosomen! Arch. f. Zellforschung, Bd. 1.

Derselbe, 1910: Zur Einigung zwischen Faden- und Granulalehre des Protoplasmas. Beobachtungen an weissen Blutzellen. Arch. f. mikr. Anat., Bd. 75 .

Derselbe, 1911, 1: Über die Beteiligung der Plastochondrien an der Befruchtung des Eies von Ascaris megalocephala. Arch. f. mikr. Anat., Bd. 76.

Derselbe, 1911, 2: Chromosomenlängen bei Salamandra, nebst Bemerkungen zur Individualitätstheorie der Chromosomen. Arch. f. mikr. Anat., Bd. 77, Abt. 2.

Derselbe, 1912: Verfolgung des sogenannten Mittelstückes des Echinidenspermiums im befruchteten Ei bis zum Ende der ersten Furchungsteilung. Arch. f. mikr. Anat., Bd. 80, Abt. 2.

Derselbe, 1913: ̈̈ber das Verhalten des plastosomatischen Bestandteiles des Spermiums bei der 'Befruchtung des Eies von Phallusia mamillata. Arch. f. mikr. Anat., Bd. 82, Abt. 2.

Derselbe, 1914, 1: Die Plastochondrien in dem sich teilenden Ei von Ascaris megalocephala. Arch. f. mikr. Anat., Bd. 84, Abt. 2.

Derselbe, 191t, 2: Verfolgung des Mittelstückes des Echinidenspermiums durch die ersten Zellgenerationen des befruchteten Eies. Arch. f. mikr. Anat., Bd. 85, Abt. 2. 
Derselbe, 1914, 3: Was sind die Plastosomen? Antwort auf die Schrift gleichen Titels von G. Retziu s. Arch. f. mikr. Anat., Bd. 85, Abt. 1 .

Derselbe, 1915: Was sind die Plastosomen? II. Bemerkungen zu dem Vortrag von C. Benda: Die Bedeutung der Zelleibstruktur für die Pathologie. Arch. f. mikr. Anat., Bd. 87. Abt. 1 (im Druck).

M a ls ow, K., 1911: Chromosomenverhältnisse bei Ancyracanthus cystidicola. Zool. Anz., Bd. 38 .

Derselbe, 1912: Der Chromosomenzyklus bei Ancyracanthus cystidicola. Arch. f. Zellforschung, Bd. 9.

v. Na a geli, C., 1884: Nechanisch-physiologische Theorie der Abstammungslehre.

Nussbaum, MI, 1906: Befruchtung und Vererbung. Anat. Anz., Bd. 28.

Pfefter, W., 1897: PHanzenphysiologie. Bd. I, Stoffwechsel. Leipzig.

Romeis, B., 1912: Benbachtungen über Degenerationserscheinungen von Chondriosomen. Nach Intersuchungen an nicht zur Befruchtung gelangten Spermien von Ascaris megalocephala. Arch. f. mikr. Anat. Bd. 80 , Abt. 2.

Derselbe. 1913: Beobachtungen über die Plastosomen von Ascaris megalocephala während der Embryonalentwicklung unter besonderer Berücksichtigung ihres Verhaltens in den Stamm- und Urgeschlechtszellen. Arch. f. milkr. Anat., Bd. 81, Abt. 2.

Rubaschkin, W, 1910: Chondriosomen und Differenzierungsprozesse bei Säugetierembryonen. Inatomische Hefte, Bd. 41.

R ï ckert, J., 1895: Über das Selbständigbleiben der väterlichen und mütterlichen Kernsubstanz während der ersten Entwicklung des befruchteten Cyclops-Eies. Arch. f. milkr. Anat, Bd. 45 .

Schaxel, J., 1911: Plasmastrulituren, Chondriosomen und Chromidien. Anat. Anz., Bd. 39.

Schoonjans, H., 1909: Etude sur la phase daccroissement des ovocytes chez Ascaris megalocephala bivalens. Bulletin de la Société Royale des Sciences médicales et naturelles de Bruxelles, no. 1.

Sobotta, J., 1913. 1: Eireifung und Befruchtung einschliesslich experimenteller Parthenogenese. Jahresber. über die Fortschritte d. Anat. a. Entwicklungsgesch.. herausgeg. von G. Schwalbe. N. F. Bd. 18, Literatur 1912, Teil 2 .

Derselbe, 1913, 2: Über eineiige Zwillinge des Menschen und die Polyembryonie bei den Gürteltieren. Sitzungsber. d. Physikal.-med. Ges. zu Würzburg, Jahrg. 1913.

Strasburger, E., 1877: Über Befruchtung and Zellteilung. Jenaische Zeitschr. f. Naturw., Bd. 11 (N. F. Bd. 4).

v. Tellyesniczky, K, 1907: Die Entstehung der Chromosomen. Evolution oder Epigenese? Berlin-Wien.

Tsukaguchi, R., 1914: Über die feinere Struktur des Ovarialeies von Aurelia aurita L. Arch. f. mikr. Anat., Bd. 85, Abt. 2.

Van Beneden, E., 1883: Recherches sur la maturation de l'oeuf, la fécondation et la division cellulaire. Archives de Biologie, vol. 4. 
Van der Stricht, 0, 1909: La structure de l'oeuf des Mammifères (Chauve-Souris, Vesperugo noctula). Troisième partie. 'L'oocyte à la fin du stade d'accroissement, au stade de la fécondation et au début de la segmentation. Mémoires publiés par la Classe des Sciences de l'Acad. Royale de Belgique, 2. sér., t. 2.

$\mathrm{Z}_{\circ \mathrm{ja}}$, L. u. R., 1891: Intorno ai plastidnli fucsinofili (bioblasti dell' $A 1 \mathrm{t}$ m a n $\mathrm{n}$ ). Mem. Ist. Lomb. Sc. Lett., Milano, vol. 16.

Zoja, R., 1896-1898: Stato attuale degli studi sulla fecundazione. Dissertazione di libera docenza. Bolletino scientifico, anno 18-20, Pavia.

\section{Erklärung der Abbildungen auf Tafel I-IV.}

Die Abbildungen der Tafel I-IV sind, nach Schnitten durch die hintersten Enden der weiblichen Geschlechtsröhren von Filaria papillosa $R$, mit $\mathrm{Z}$ eis s 'Apochromat $1,5 \mathrm{~mm}$ und Kompensationsokular 12 unter Benutzung des $\mathrm{A}$ b beschen Zeichenapparates entworfen, wobei der Abstand ler Zeichenebene von der Ebene des 'Tisches $17 \% \mathrm{~cm}$ betrug

\section{Tafel I.}

Fig. 1-25̆. Sämtlich nach Präparaten, welche mit modifiziertem Flem$\mathrm{m}$ in gschem Gemisch fixiert sind.

Fig. 1-10. Freie Spermien aus dem obersten Abschnitt des Uterus.

Fig. 1-5. Färbung mit Safranin. Chromatinkügelchen rot, Nebenkern etwas stärker bräunlich gefärbt als das Cytoplasma. In Fig. 2 und 3 sind sechs, in Fig. 1, 4,5 fünf Chromatinkügelchen zühlbar. Von den fünf Kügelchen in Fig. $\overline{\mathbf{5}}$ ist eines deutlich grösser als die ïbrigen, setzt sich also wohl aus zweien zusammen.

Fig. 6-10. Färbung mit Eisenhämatoxylin. Nebenkern intensiv schwarz gefärbt. Die gleichfalls schwarz gefärbten Chromatinkügelchen sind in Fig. 6 durch den Nebenkern verdeckt, in den Figuren 7-10 dagegen sichtbar, aber mehr oder weniger untereinander verlilumpt.

Fig. 11-26. Färbung mit Eisenhämatoxylin.

Fig. 11-17. Verschiedene Entwicklungsstadien von Oozyten. Text S. 21-23.

Fig. 18-26. Befruchtete Eizellen. Eiplastochondrien infolge stärkerer Differenzierung nicht mehr schwarz wie in Fig. 17. sondern nur noch grau gefärbt.

Fig. 18, 19. Spermium eben eingedrungen, unmittelbar unter der Zelloberfläche gelegen. Text S. 24-25.

Fig. 20-26. Das Spermium liegt zwischen dem Eikern und dem in den Figuren oberen Pol der Eizelle. Auswanderung der männlichen Plastochondrien. Text S. 27-29.

Über die Vakuole. welche sich in Fig. 23, 25 und 26 am Rand des Spermiums findet. s. Text S. 31. 


\section{Tafel II.}

Sämtliche Figuren nach Präparaten, welche mit modifiziertem Flem ming schen Gemisch fixiert und mit Eisenhämatoxylin gefärbt worden sind. Fig. 27-35. Weitere Entwicklungsstadien befruchteter Eizellen.

Fig. 27-31. Die Auswanderung der männlichen Plastochondrien aus dem Spermienkörper nimmt ihren Fortgang.

Von Fig. 28 an: Beginn der ersten Reifungsteilung. Der Eikern verlässt den Eimittelpunlit und begilot sich an den in den Figuren unteren Pol der Zelle. Fig. 31 Eikern verkleinert. Fig. 32 Membran des Eikerns geschwunden, Chromosomen zu einem Haufen rersammelt. Fig. 33, 34 Erste Richtungsspindel auf der Höhe der Ausbildung. Fig. 33 Erste Richtungsspindel in Seitenansicht. Fig. 34 Chromosomen der ersten Richtungsspindel in Polansicht.

In Fig. 32 sind in Eicytoplasma noch ca. 15, in Fig. 33 noch cat. 8 Körner vorhanden, welche etwas grösser und stïrker gef:ïrht als die übrigen sind.

Fig. 34. Das Eicytoplasma ist ausschliesslich ron kleinen Körnern von ter Grösse und Fürbbarkeit der Eiplastochındrien durchsetzt.

Fig. 35̃. Erste Furchungsspindel auf der Höhe der Ausbildung. Buschaffenheit des Cytoplasmas wie in Fig. 34. Im unteren Pol der Zelle die beiden Richtungskörper.

Die in den Figuren 27, 29 und 31 am Rande des Spermienkörpers gelegene Vakuole ist im Text $S .31$ besprochen: ebendort das Kügelchen, welches in Fig. 33 oberhalb des Spermienkürpers liegt; in letzterem vier Chromatinkügelchen.

Mlit Bezug auf den grossen, annïhernd oralen, hellen Bezirk (mit darin eingeschlossenem Körperchen), welchen man in Fig. 27, 28. 30, 32--34 an obern $\mathrm{Pol}^{\prime}$ der Eizelle wahrnimmt, vergl. Text S. 27-28, über den gleichen Bezirk in Fig. 26, siehe Text S. 28, Anm.

Fig. 36. Zweizellenstadium. Das Cytoplasma enthält ausser den Plastochondrien grau gefïrbte Ballen von homogenem Aussehen.

Fig. 37. Furchungsstadium, acht Zellen auf dem Schnitt getroffen. Beschaffenheit des Cytoplasmas wie in Fig. 36.

Fig. 38. Stark vorgerücktes Furchungsstadium, ca. 70 Zellen auf dem Schnitt getroffen. Ihr Cytoplasma enthält dicke Plastokonten, welche in einer homogen aussehenden Grundsubstanz eingebettet sind.

\section{Tafel III.}

Sämtliche Figuren nach Präparaten, welche mit Sublimat-Alkohol-Eisessig tixiert und mit Giems a lösung gefärbt worden sind.

Fig. 39-48. Freie Spermien aus dem obersten Abschnitt des Uterus.

Fig. 39-42. Das ganze Spermium mit Ausnahme der Chromatinkügelchen ist in Fig. 39-41 rotviolett, in Fig. 42 blauviolett gefürbt. Nan zählt in Fig. 39 und 42 sechs, in Fig. 41 fünf, in Fig. 40 dagegen nur vier Chromatinkügelchen: von den letzteren sind wahrscheinlich mindestens zwei verklumpt. 
Mitwirkung der Plastosomen bei der Befruchtung des Eies.

Fig. 43. Kopfkappe rotviolett, das iibrige Spermium mit Ansnahme der (fünf) Chromatinkügelchen blauviolett gefürbt.

Fig. 44. Kopfliappe rotviolett, Nebenkern und Schwanzteil des Spermiums blauviolett gefärbt. Chromatinkügelchen (fünf ?) verbacken.

Fig. 4ó. Kopflappe und Schwanzteil des Spermiums rotviolett, Nebenkern bläulich gefärbt. Man zählt vier Chromatinkügelchen.

Fig. 46-48. Kopflappe rotriolett, Nebenkern bläulich, Schwanzteil des Spermiums blanviolett gefürbt. In Fig. 46 sind sechs, in Fig. 47 und 48 je fünf Chromatinkügelchen zählbar. In Fig. 48 erscheint die Kopflkappe zu einer Spitze ausgezogen; wahrscheinlich handelt es sich bei dieser Spitze um ein Kunstprodultt.

Fig. 49-62. Befruchtete Eizellen.

Fig. 49-51. Spermium eben eingedrungen, unmittelbar unter der Zelloberfläche gelegen. In Fig. 49 sind die Chromatinkügelchen des Spermiums bis auf eines durch die intensive Blaufärbung des Spermiencytoplasmas verdeckt. In Fig. 50 und 51 ist die Blaufärbung. verblasst; die Chromatinkïgelchen, in Fig. 50 fünf, in Fig. 51 sechs, sind deutlich sichtbar yeworden. In Fig. 50 und 51 nimmt min ausserdem noch in dem abgeblassten Spermiencytoplasma ein stärker blau gefärbtes Körperchen unbekannter Natur wahr.

Fig. 52-58. Spätere Stadien befruchteter Eizellen. vor Beginn der ersten Reifungstcilung. Das Spermium hat seine oberflächliche Lage aufgegeben und seinen Platz zwischen dem Eikern und dem in den Figuren oberen Pol der Eizelle eingenommen. In Fig. 52, 5:3, 5̃ und 56 ist die Blaufürbung des Spermiencytoplasmas abgeblasst, in Fig. 54, 57 und 58 dagegen erhalten. Fig. 52-56 zeigen das Grösserwerden der Chromatinkügelchen. In Fig. 52-5̃ zählt man sechs, in Fig. 56 fünf oder sechs, in Fig. 57 drei untereinander verbackene, in Fig. 58 vier Chromatinkügelchen.

Fig. ว9. Eikern im Beginn der ersten Reifungsteilung. Spermienkörperschalenförmig umgestaltet; auf dem optischen Schnitt: fünf Chromatinkügelchen.

Fig. 60. Membran des Eikerns geschwunden, Chromosomen der ersten Richtungsspindel. Im Eicytoplasma zwei stärker gefärbte rundliche Flecke, deren ich im Text keine Erwähnung getan habe; Natur derselben mir umbekannt. Im Spermienkörper drei Chromatinkiigelchen sichtbar.

Fig. 61. Eizelle kurz vor Ausstossung des ersten Richtungskörpers. Rest des Spermiencytoplasmas mit fünf Chromatinkügelchen.

Fig. 62. Beide Richtungskörper ausgestossen. Männlicher und weiblicher Vorkern. In letzterem vier Chromatinklumpen; am oberen Rand des ersteren ein strangförmiger Rest des Spermiencytoplasmas.

\section{Tafel IV.}

Sämtliche Figuren nach Präparaten, welche mit modifiziertem Flemming schen Gemisch fixiert und mit Eisenhämatoxylin gefärbt worden sind. Die Strukturverhältnisse des Eicytoplasmas sind nicht wiedergegeben. 
Fig. 63-77. Spätere Entwicklungsstadien befrnchteter Eizellen.

Fig. 63 und 64. Erste Richtungsspindel; Chromosomen derselben in Fig. 63 wenig, in Fig. 64 weiter auseinandergewichen. Chromatinkügelchen des Spermiums blass gefärbt.

Fig. 65-68. Erster Richtungskörper ausgestossen.

Fig. 6ã. Chromatinkügelchen des Spermiums stärker fürbbar geworden.

Fig. 66. Chromatinkügelchen des Spermiums zum Teil zelschnürt.

Fig. 67. Kleiner männlicher Vorkern von unregelmässiger Form.

Fig. 68. Chromatinkügelchen des Spermiums auf drei Hüufchen verteilt. welche durch Zwischenrïume voneinander getrennt sind.

Fig. 69-7t. Beide Richtungskörperchen gebildet.

Fig. 69. Männlicher Vorkern grösser als in Fig. 67, aber noch unregelmässig in der Form. Die im Ei zurückgebliebenen Chromosonen der zweiten Richtungsteilung haben sich noch nicht zum weiblichen Vorkern umgebildet.

Fig. 70. $Z$ w e i männliche und ein weiblicher Vorkern.

Fig. 71. Ebenso: die Vorkerne etwas grösser geworden.

Fig. 72, 73. Je e in männlicher und weiblicher Vorkern: in letzteren sind in Fig. 72 die Chromosomen der zweiten Richtungsteilung noch in Form von Chromatinklumpen erhalten.

Fig. 7t. Z w ei männliche (oben) und ein weiblicher Vorkern (unten).

Fig. 75. In den Vorkernen sind wieder Chromosomen aufgetreten.

Fig. 76, 77. Die Chromosomen der ersten Furchungsspindel in Polansicht: man zählt in Fig. 76 elf, in Fig. 77 zwölf Chromosomen.

Über die anniihernd ovalen, hellen Bezirke (mit darin eingeschlossenen Körperchen), welche man in Fig. 63 und 64 am oberen $P_{0}$, in Fig. $65-67$ an den Seiten der Eizelle (in Fig. 66 in doppelter ZahI) wahrnimmt, s. Text S. 28; bezüglich des homogen aussehenden Kügelchens, welches in Fig. $6 \dot{5}$ in der Nachbarschaft der Chromatinkügelchen des Spermiums, in I'ig. 69-71 in der Nähe des männlichen Vorkerns, in Fig. 72 links vom weiblichen gelegen ist, ist Text $S .31$ zu vergleichen. 


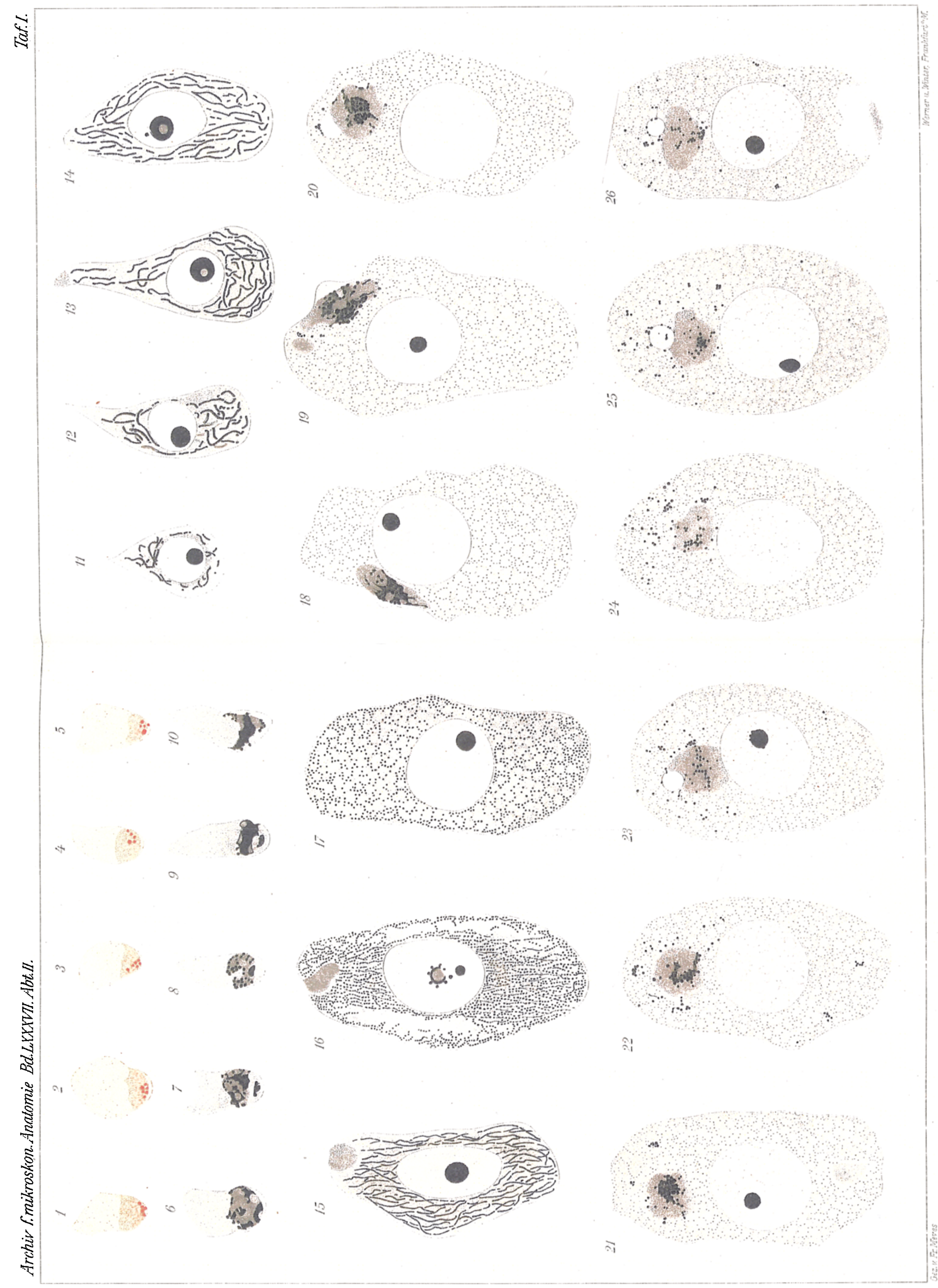




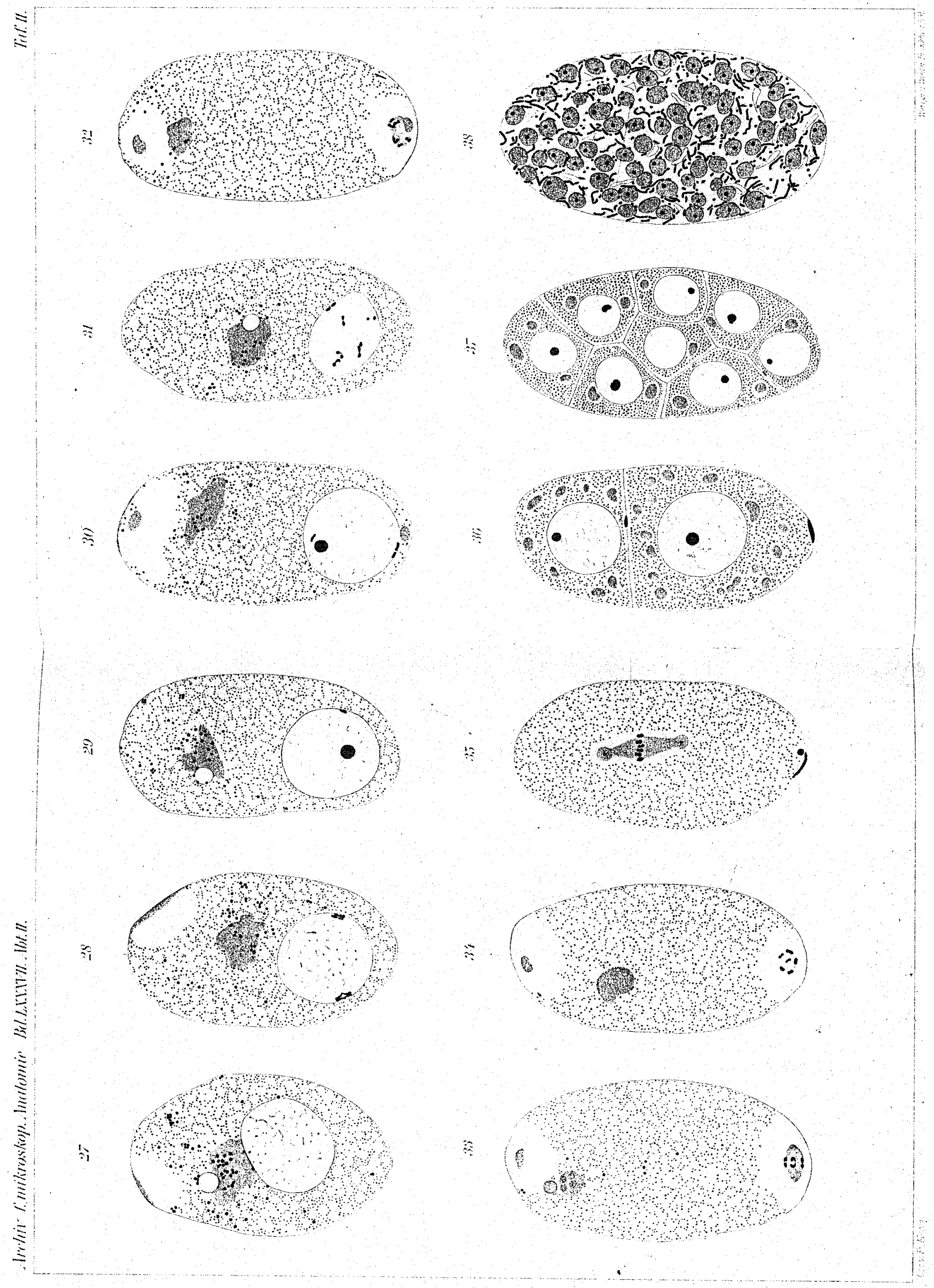




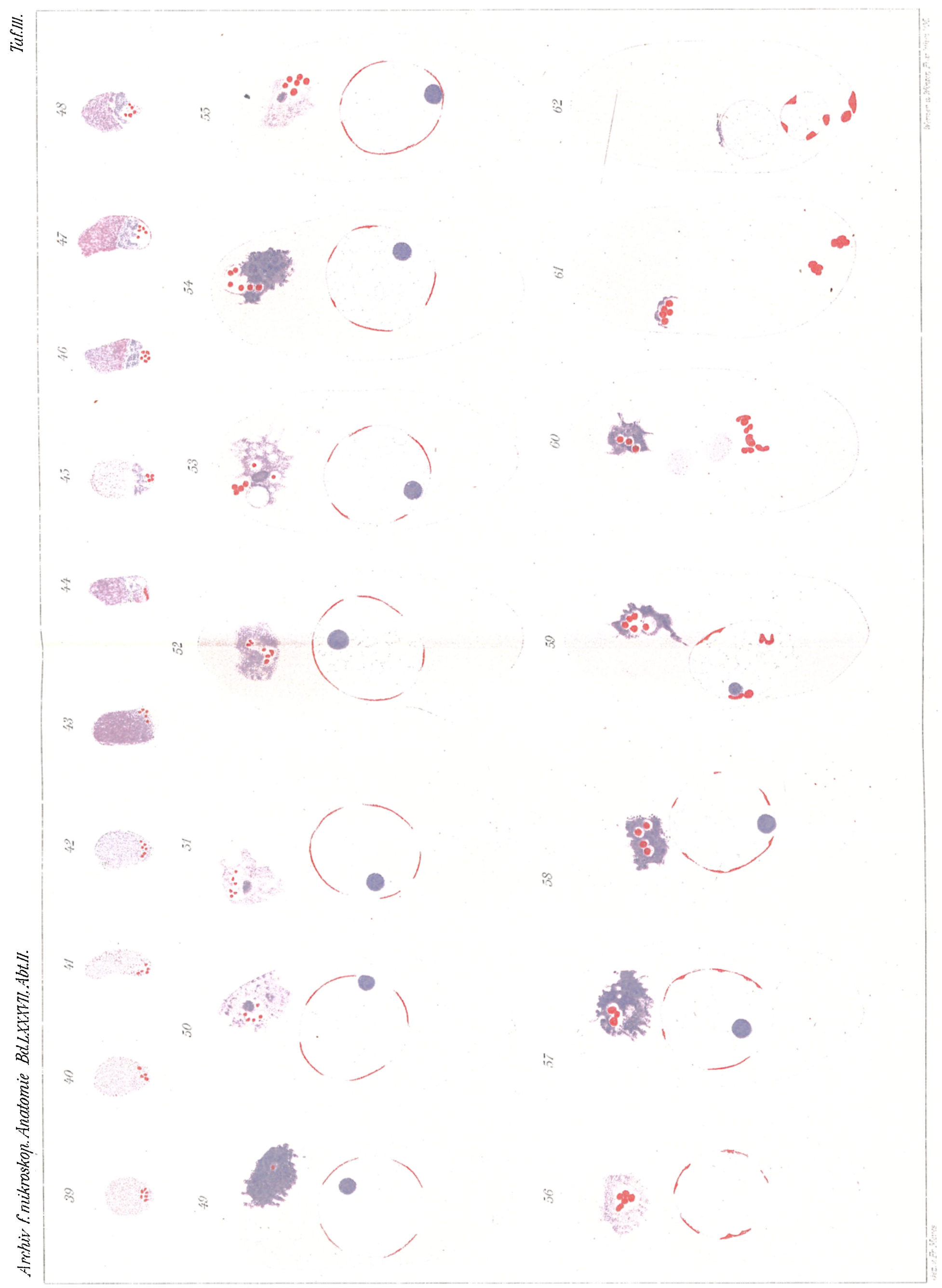


